\title{
FINE-GRAINED TYPE-FREE INTENSIONALITY
}

Commonplace syntactic constructions in natural language seem to generate ontological commitments to a dazzling array of metaphysical categories - aggregations, sets, ordered $n$-tuples, possible worlds, intensional entities, ideal objects, species, intensive and extensive quantities, stuffs, situations, states, courses of events, nonexistent objects, intentional and discourse objects, general objects, plural objects, variable objects, arbitrary objects, vague kinds and concepts, fuzzy sets, and so forth. But just because a syntactic construction in some natural language appears to invoke a new category of entity, are we theoreticians epistemically justified in holding that there are such entities? This would hardly seem sufficient. To be epistemically justified, the ontology to which we theoreticians are committed must pass strict standards: the entities must be of the sort required by our best comprehensive theory of the world. The thesis of this paper is that fine-grained type-free intensional entities are like this. If the thesis is right, these entities have a special objective status perhaps not possessed by some of the other ontological categories associated with special syntactic constructions in natural language. In fact, it is plausible to hold that fine-grained typefree intensional entities provide the proper minimal framework for constructing logical and linguistic theories. In this paper my strategy will be to survey the competing conceptions of fine-grained type-free intensionality and to present arguments in support of one of them. Following this narrowing down process, I will go on to the indicated epistemological considerations.

\section{FROM INTENSIONAL LANGUAGE TO INTENSIONAL ENTITIES}

We begin by isolating two central uses of the term 'intensional', one logico-linguistic and one ontological. First, the logico-linguistic use. A language is extensional if, within it, equivalent formulas can be substituted for one another salva veritate:

$$
(A \leftrightarrow B) \rightarrow(\ldots A \ldots) \leftrightarrow(\ldots B \ldots) .
$$

Gennaro Chierchia, Barbara H. Partee, and Raymond Turner (eds.), Properties, Types and Meaning, I, 177-230.

(c) 1989 by Kluwer Academic Publishers. All rights reserved. 
A language is intensional if this substitutivity principle does not hold for it.

Second, the ontological use. An item $x$ is an extensional entity if it satisfies all relevant principles of extensionality. Principles of extensionality come in two forms. First, let ' $R$ ' be any copula ('is', 'is an instance of', 'is related by', 'is a member of', 'is a part of', 'has', 'falls under', etc.) and let the item $x$ be in the same ontological category as some item in the range of $R$. Then for all $y$ in the same ontological category as $x$ :

$$
(\forall z)(z R x \leftrightarrow z R y) \rightarrow x=y .
$$

Second, let ' $T$ ' be any verifier ('is true', 'is so', 'obtains', 'holds', 'happens', 'occurs', etc.) and let the item $x$ be in the same category as some item that is an instance of $T$. Then for all $y$ in the same category as $x$

$$
(T x \leftrightarrow T y) \rightarrow x=y .
$$

With this definition of extensional entity we may define intensional entity: an item $x$ is intensional if it is not extensional. That is, an item $x$ is intensional if it fails to satisfy one or more of these extensionality principles.

Paradigmatic intensional entities are properties, relations, and propositions (PRPs). For example, suppose that $x$ is the property of being a round square. There is an item (e.g., any non-null property) that is in the same ontological category as $x$ and that is in the range of the relation expressed by the copula 'has'; and there is an item (e.g., the property of being the thirteenth Apostle) that is in the same ontological category as $x$ and that is had by all and only those items had by $x$ and that is nevertheless not identical to $x$. Therefore, $x$ fails to satisfy a principle of extensionality and, hence, is an intensional entity. For another example, suppose that $x$ is the proposition that $5+7=13$. There is an item (e.g., any true proposition) that is in the same ontological category as $x$ and that is an instance of the property expressed by the verifier 'is true'; and there is an item (e.g., the proposition that grass is red) that is in the same ontological category as $x$ and that is true if and only if $x$ is true and that is nevertheless not identical to $x$. Therefore, $x$ fails to satisfy a principle of extensionality and, hence, is an intensional entity. Sets, by contrast, are extensional entities. To illustrate, let $x$ be some set, either null or non-null. There is an item (i.e., any non-null set) that is in the same ontological category as $x$ and that is in the range of the copula 'is a member of'. So the principle of extensionality relevant to $x$ is the one stated in terms of "is a member of'. However, there is no item (besides $x$ itself) that is in the same ontological category as $x$ and that has for members exactly the items that are members of $x$. So $x$ satisfies this principle of extensionality and, thus, is an extensional entity.

A leading way of trying to justify a theory of properties, relations, and propositions is to show that it provides the best background theory in which to develop a theory of language, specifically, a theory of the semantics of natural languages. Advocates of this approach hold that predicates, verb phrases, and open-sentences express properties or relations and that sentences express propositions. But many people (Quine, Davidson, and others) are skeptical that these bits of language really express anything at all because they are skeptical that there is any such thing as meaning above and beyond what can be captured by a theory of reference and truth. Supporters of PRP semantics are undaunted by this skepticism because they think PRPs are in any event needed for giving a theory of reference and truth, and as long as PRPs are needed there, it would be pointless not to use them in a theory of meaning. The reason PRPs are needed in a theory of reference and truth is that there are special syntactic constructions - 'that'-clauses, gerundive phrases, infinitive phrases, and the like - that behave as singular terms, and to give the truth-conditions for sentences containing these singular terms, which are called intensional abstracts, we seem forced to assign references to them. When we do so, PRPs are the simplest and most natural choice because (except in certain problem cases) their identity conditions mirror beautifully the intensional substitutivity conditions on expressions occurring within intensional abstracts. This is the meeting of the ontological and logico-linguistic uses of 'intensional': the identity conditions of these intensional entities mirror the substitutivity conditions of the intensional abstracts that denote these entities. (This line of argument was developed in detail in my book Quality and Concept.)

There is a growing consensus among philosophers, logicians, and linguists that this match-up between intensional entities and intensional abstracts establishes two prominent features. First, PRPs are at least in some cases very fine-grained entities. The argument is that the substitutivity conditions governing the intensional abstracts that denote 
these PRPs are very fine-grained. (I will return to this argument for fine-grainedness later.) Because the distinctions among these PRPs are so fine, we may without being too misleading talk as though the PRPs denoted by syntactically complex intensional abstracts are themselves "complex" entities. In the same vein, we may say that these complex PRPs have "constituents" that are associated with relevant syntactic constituents of the denoting intensional abstracts. (I should emphasize that on the algebraic approach developed in Quality and Concept this talk of "complexity" and "constituency" is not to be taken as literal part/whole talk; it is heuristic only.) The second prominent feature is that (a theory of) PRPs may be expected to be type-free. But what does 'type-free' mean? This is the first question I will investigate.

\section{SIX TYPES OF TYPE-FREEDOM}

There has been a good bit of terminological confusion in recent discussions of this matter. ${ }^{1}$ There are at least six independent notions of type-freedom, each of which is disallowed by classical type theorists such as Russell and Church. It would be good to begin by spelling them out briefly.

(1) Transcendental predicates. The first way in which a theory of PRPs may be said to be type-free is for it to contain transcendental predicates (in the Aristotelian sense), that is, predicates that apply freely across metaphysical categories: for example, predicates like 'contemplate', 'think of', 'is self-identical', 'is some kind of item or other', and so forth. ${ }^{2}$

(2) Universal universal quantification. To say the general things we would like to say with transcendental predicates (e.g., 'For all $x, x=x^{\prime}$ ), we need a sort of variable (or similar device) whose values range freely over the entire universe of discourse. The second way in which a theory may be said to be type-free is for it to contain variables like this and quantifiers to go with them. In this case, we may call the universal quantifier a universal universal quantifier.

(3) Impredicative intensional abstracts. A third type of type-freedom concerns the kinds of quantifier phrases - specifically, the quantified variables - that are permitted to occur within intensional abstracts. The theory may be called type-free if the values of these quantified variables include the very PRP denoted by the intensional abstract itself. For example, the following intuitively valid sentence contains an example of this kind of quantification within an intensional abstract:
That Descartes doubts everything implies that Descartes doubts that Descartes doubts everything.

In symbols,

$$
[(\forall x) D d x] \text { Implies }[D d[(\forall x) D d x]] .
$$

Formulas containing quantifiers like this were called impredicative by Russell, and they are, of course, ruled out in ramified type theories like those of Russell and of Church.

(4) Self-embedded predicates. The fourth type of type-freedom is also illustrated by this example. Consider the second intensional abstract ' $[D d[(\forall x) D d x]]$ '. The first occurrence of the predicate 'doubts' takes as an argument the intensional abstract ' $(\forall x) D d x]$ ' which contains the very same predicate 'doubts' once again. Now one might think that this kind of self-embedding is only an appearance, specifically, that the two superficially similar predicates ('doubts' and 'doubts') are really semantically distinct. But Davidson has argued that human beings can learn only a finite number of semantically primitive constants and therefore, that in an ideal representation of the syntax of natura languages there should also be a finite number of semantically primitive constants. Because verbs like 'doubts' can be self-embedded any (finite) number of times, the only way to meet Davidson's finiteness requirement is to hold that the supply of semantical primitives must eventually give out. Consequently, once a certain depth of embedding is reached, all more deeply embedded occurrences of the predicate 'doubts' mus be semantically the same as some earlier one. Hence, genuinely selfembedded occurrences are unavoidable. Given a PRP semantics, therefore, there will be propositions that contain as constituents a relation of doubting that is predicated of a proposition that contains as a constituent the very same relation of doubting. In this sense we may say that, not only is the predicate 'doubt' self-embeddable, but also the relation of doubting is self-embeddable. So, for example, we get decomposition trees like this:

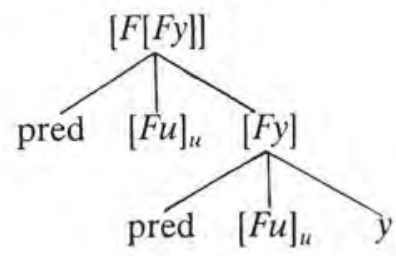


where pred is the operation of singular predication. (The intensional abstract ' $[\mathrm{Fu}]_{u}$ ' denotes the property of being an item $u$ such that $F u$. Incidentally, it is quite wrong to use $\lambda$-notation for PRPs. The $\lambda$ notation has a well-established use for functional-abstraction. So using it for PRP abstraction invites conceptual confusion. Properties are very different from functions, including propositional functions. And propositions intuitively bear no resemblance at all to functions. Much more will be said on this later. To avoid this confusion with functions, I use my bracket-notation to denote PRPs.)

(5) Self-constituency. A fifth way in which PRPs could be type-free would be for PRPs to be not just self-embedded but also constituents of themselves. This is quite a different affair, which would occur if in a decomposition tree a node could occur on a path descending from itself:

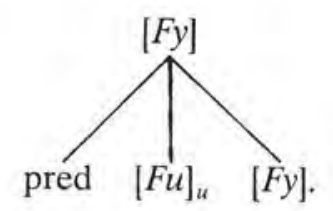

Perhaps among the things I perceive when I look in a mirror is an item $y$ such that $y=[$ I see $y]$. If so, $y$ would have a decomposition tree like this:

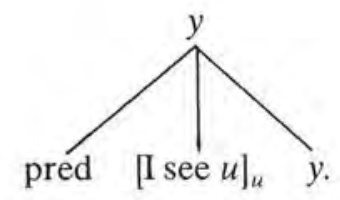

Or if the sentence 'I think this' [i.e., this very thought itself] expresses a proposition (that is, if this $=$ the proposition that I think this), it would have a decomposition tree like the following:

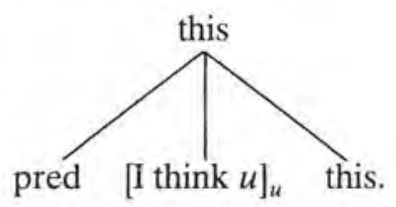

If the Liar sentence ('This is false') or the Truth-teller sentence ('This is true') express propositions, these propositions would have the following structure:
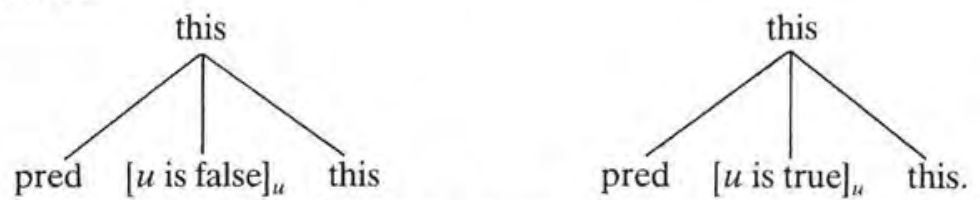

These trees are non-well-founded inasmuch as some of their branches do not terminate but instead cycle. PRPs with non-well-founded decomposition trees may be called type-free for the following reason. If a wellfoundedness condition were imposed on PRP-decomposition trees, it would induce a type structure. Specifically, higher-level nodes can be built from lower level nodes but not vice versa; therefore, one could say that higher level nodes are of a higher type than their lower level nodes. Such a type structure is built into in ramified type theories like those of Russell and of Church. But it is absent if non-well-founded PRP-trees are allowed. (This type of type freedom might bring to mind the way in which the type structure implicit in the iterative hierarchy is absent in a non-well-founded set theory, but it is really a very different logical phenomenon. Some situation semanticists have tried to treat the former type of type freedom as a special case of the latter. I will show why this is confused in a moment.)

(6) Self-instantiation. A sixth way in which a PRP theory can be type-free arises in connection with predication relations. Predication relations are expressed in natural language by the copulas appropriate to properties and relations, that is, verb phrases such as 'is', 'is an instance of', 'is related by', 'has', 'falls under', and so forth. [The relation implicit in higher-order "sentences" like 'runs(runs)' and 'red(red)' and higher-order "open-sentences" like ' $x(x)$ ' may also be counted as a predication relation.] The theory would be type-free if it contains true sentences or theorems asserting the existence of properties that are instances of themselves (or instances of instances of themselves, or the like). Being self-identical has the property of being self-identical; being liked by someone has the property of being liked by someone; and so forth. In theories of this sort, one often asserts the existence of a universal property, that is, a property of which everything is an instance including the universal property itself. Of course, as soon as one permits self-instantiation, one gets a host of difficult problems. Not only 
Cantor-style, Burali-Forti-style, and Russell-style paradoxes, but also philosophically loaded questions such as: what is the cardinality of the property of being uniquely self-instanced, i.e., $[u$ is the unique instance of $u]_{u}$ ? And once self-instantiation is permitted, one also wonders whether there could be any good reason not to require every satisfactory PRP theory to provide all of the properties needed to validate Frege's highly intuitive analysis of number. (Such a theory would have to posit the property of being a property with one instance, the property of being a property with two instances, and so forth.)

Now in Russellian or Churchian ramified type theories there are type restrictions ruling out each of these six types of type-freedom: no transcendental predicates, no universal universal quantification, no impredicative intensional abstracts, no self-embedding, no self-constituency, and no self-instantiation.

When I wrote Quality and Concept, one of my goals was to develop a framework for PRP theories that could be type-free in each of these six ways. Let me summarize briefly what my strategy was. (1) The way to model transcendental predicates is to begin with a single infinite domain in which all categories of entities - PRPs and non-PRPs alike - are treated on a par as primitive entities. Accordingly, when we assign an extension to a predicate the extension may include objects in any metaphysical category in this unitary domain. (2) Universal universal quantification is then automatic; we simply have variables whose range of values is the whole domain. (3) PRPs in the domain are related to one another algebraically. Specifically, PRPs denoted by complex intensional abstracts are the result of applying appropriate logical operations (conjunction, negation, existential generalization, singular predication, etc.) to relevant PRPs and nonPRPs in the domain. Impredicativity is then achieved simply by setting up the domain so that in the appropriate way it is closed under these logical operations, in particular, under the operation of generalization. (4) Likewise, selfembedding is achieved as a byproduct of the fact that in the appropriate way the domain is closed under the operation of predication. Singular predication (pred, for short) is an operation that takes $n$-ary intensions $\left[R u_{1} \ldots u_{n-1} u_{n}\right]_{u_{1} \ldots u_{n-1} u_{n}}$ (for $n \geqslant 1$ ) and items $x$ to $n-$ 1 -ary intensions $\left[R u_{1} \ldots u_{n-1} x\right]_{u_{1} \ldots u_{n-1}}$. In the limiting case, then, pred would take, say, the relation of liking and the relation of liking to the property of liking the relation of liking:

$$
\operatorname{pred}\left([u \text { likes } v]_{u v},[u \text { likes } v]_{u v}\right)=\left[u \text { likes }[u \text { likes } v]_{u v}\right]_{u} \text {. }
$$

This illustrates how self-embedding is achieved simply by closing the domain under the operation pred. (5) Self-constituency is achieved just by permitting decomposition trees in which PRPs occur on paths descending from themselves. In Quality and Concept, there is a fully developed theory for PRPs with self-constituency, namely, the theory for PRPs on the coarse-grained conception (according to which necessarily equivalent PRPs are identical). According to this conception, every PRP has infinitely many non-well-founded decomposition trees. Consider an example. Because $[F x]_{x}$ and $\left[F x \&[F x]_{x}=[F x]_{x}\right]_{x}$ are necessarily equivalent properties, they are identical on the coarsegrained conception. So the property $[F x]_{x}$ (i.e., $\left[F x \&[F x]_{x}=[F x]_{x}\right]_{x}$ ) has a decomposition tree in which $[F x]_{x}$ occurs on a path descending from itself:

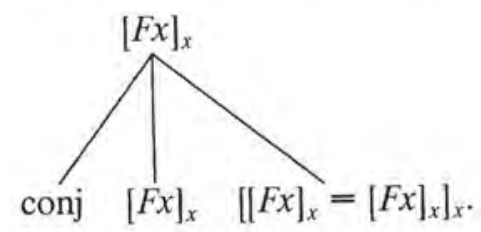

By the same token, one can model self-constituency on various finegrained conceptions of PRPs. To achieve this, one needs no revisions in the background set theory (as is the case in situation semantics, for example). All that is needed is to set up the model with one trivial feature: the logical operations are permitted to cycle. Non-well-founded operations like this are commonplace in all familiar well-founded set theories. For example, the operation that takes integers $x$ to $-x$ and $-x$ back to $x$. This operation yields non-well-founded trees:

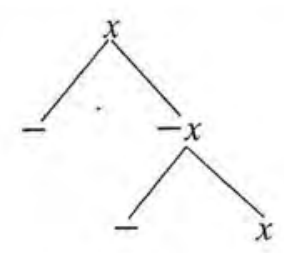

No revisions in the background set theory are needed. 
The first five types of type-freedom are thus unproblematic on the Quality and Concept approach. Before turning to the sixth type of type freedom, I will say a few words about alternate approaches to the first five types of type freedom. First, the standard extensionalist approach. This approach to modeling PRPs is to begin with a domain of nonPRPs (usually just particulars) and then to try to construct PRPs from these items by means of various set-theoretic operations. The possibleworlds approach is an example of this approach; it is distinctive only because it begins with a domain of individuals most of which are not actual. (This is something I do not favor philosophically. I am inclined to accept realism in the primary sense defined by Alonzo Church: namely, that there are only real things, that there are no unreal things. To me it seems intuitive that whatever is real is actual.) The kind of categorial semantics originally formulated by Richard Montague is an example of the standard extensionalist approach to modeling PRPs inasmuch as it is based on the possible-worlds method. (The theory developed by M. Cresswell in Structured Meanings is a special hybrid of the standard extensionalist approach, and it has each of the difficulties that I describe below.)

However, the standard extensionalist approach is beset with difficulties. In the present paper I will mention only those difficulties having to do with type freedom. Specifically, it is unable to represent transcendental predicates, self-embedding, and self-constituency. For example, on this approach there will be no (set-theoretic surrogate for the) transcendental, self-embedded relation of liking. In this connection, there will be no (set-theoretic surrogate for the) proposition that someone likes the relation of liking. (In symbols, $\left[(\exists x) x\right.$ likes $\left.[u \text { likes } v]_{u v}\right]$.) Moreover, there will be no (set-theoretic surrogate for the) transcendental, self-embedded relation of identity; nor is there a (set-theoretic surrogate for the) proposition that identity is identical to identity. (In symbols, $\left[[x=y]_{x y}=[x=y]_{x y}\right]$. .) The only way for advocates of the standard extensionalist approach (possible-worlds theorists, original Montague-style categorial semanticists, etc.) to compensate is to resort to a non-standard set theory that posits ill-founded "sets": $x \in \ldots \in x$. This, I will argue in a moment, is too a big price for anyone to pay at this stage of research.

Now in situation semantics, by contrast to the standard extensionalist approach, transcendental properties and self-embedded properties can be modeled without resorting to ill-founded "sets" because, just as on the algebraic approach developed in Quality and Concept, properties and relations are taken at face value as irreducible entities. (In what follows, each of my criticisms applies not only to situation semantics but also to the approaches taken in Scott Soames' "Direct Reference and Propositional Attitudes" and Nathan Salmon's Frege's Puzzle.) But for some unexplained reason, situation semanticists go only half way: still under the spell of the reductionistic urge of the standard extensionalist approach, situation semanticists (and Soames and Salmon) try to use set theory to construct situations (and propositions) and complex properties and relations out of basic properties and relations and individuals. For example, situation semanticists identify situations with ordered sets of basic properties and relations and individuals, or they identify situations with partial functions from times to such ordered sets. Thus, just as with the standard extensionalist approach - e.g., the possible-worlds approach or the original Montague-style categorial semantics - these "semi-reductionistic" approaches would have us believe that situations (and propositions) and complex properties and relations are ordered sets or functions.

I will state three basic problems with these "semi-reductionistic" approaches. First, is it at all plausible that, when a person sees that the glass is half full, the person is perceiving an ordered set or a function? People who hold this kind of thing have lost their "semantic innocence." If, instead, we take all intensions at face value as irreducible entities either basic qualities, basic relations, or nonbasic items resulting from logical combinations of basic items - these counterintuitive consequences are avoided. Situations could thus be easily accommodated within our PRP theory. Indeed, a satisfactory situation theory is just a component of any comprehensive PRP theory.

The second problem with these semi-reductionistic approaches (i.e., the approaches of Barwise and Perry, of Soames, and of Salmon) is that they take coarse-grained properties and relations as given entities without providing any theory for them. Coarse-grained PRPs form that natural family of intensions that are identical whenever they are necessarily equivalent. Consider shapes, for example. The shapes trilateral and triangular are necessarily equivalent, and intuitively, they are one and the same shape. Likewise, for all other qualities: they are identical if necessarily equivalent. Basic relations (connections, as I call them) are like this, too. For example, if knowing and having a justified true belief are necessarily equivalent relations, then knowing just is the same thing 
as having a justified true belief. And it seems that situations in nature are also like this. The lake's being half full and lake's being half empty are, intuitively, the same natural situation (condition, state of affairs, phenomenon). (Of course, the concept of being trilateral $\neq$ the concept of being triangular; the concept of knowing $\neq$ the concept of having a justified true belief; the thought that the lake is half full $\neq$ the thought that the lake is half empty. Concepts and thoughts are fine-grained intensions.) How would semi-reductionists formulate a theory of coarsegrained intensions (and of coarse-grained intensional abstraction)? The possible-worlds approach is unsatisfactory, as we have seen. An algebraic approach is the only viable approach known. (For reasons I will give below, a propositional-function approach is unsatisfactory.) But if an algebraic approach is needed here, there can be no good reason for not using an algebraic approach to treat fine-grained intensions as well. By identifying fine-grained intensions with set-theoretic constructs (ordered sets, partial functions, etc.) and by treating coarse-grained intensions algebraically, semi-reductionists would end up with a disunified, ad hoc theory. By contrast, if one uses an algebraic approach to treat both fine-grained and coarse-grained intensions, the result is a unified, natural theory.

The third problem with the semi-reductionistic approaches concerns self-constituency. When situation semantics tries to model this phenomenon, it loses a primary gain it made over the standard extensional approach. We saw that, to model transcendental predicates and self-embedding, the standard extensional approach (e.g., the possibleworlds approach) was forced to invoke a non-well-founded "set" theory. By taking properties and relations at face value as irreducible entities, semi-reductionists are able to avoid this theoretically unwise move. But to model self-constituency, semi-reductionists are themselves forced to invoke a non-well-founded set theory. So what once seemed to be a gain over the standard extensionalist approach is lost if self-constituency is incorporated: non-well-founded set theory must be adopted.

There are two grave problems with adopting this radical theoretical architecture. First, according to the majority of set theorists, it is just logically impossible for sets (in contradistinction properties) to be nonwell-founded. To these set theorists, talking of non-well-founded sets is just a category mistake, like talking of objects with shapes but no size. Second, and methodologically more serious, this move forces one at this very early, mathematically elementary stage of inquiry to take a stand on how ultimately to solve the set-theoretical paradoxes. But methodologically this is a grave mistake. In the foreseeable future it is highly unlikely that we are really going to understand the pathology of these paradoxes or that we are really going to know what form an ideal resolution of them should take. It would be hubris to think otherwise. So an approach that unnecessarily forces us into taking a stand on these insolubilia is decisively less desirable than an approach that does not force us to do this.

For this reason, therefore, our nonreductionistic algebraic approach to PRPs is superior. Because we do not identify fine-grained intensions with sets but instead take them at face value as irreducible entities, we can straightforwardly model self-constituency without adopting any position on how to resolve the set-theoretical paradoxes; we need only have decomposition trees with nonterminating branches, a commonplace in any set theory.

Summing up. On grounds of intuition, theoretical unity, and methodology, our algebraically motivated PRP theory provides a decisively better way to represent the first five types of type-freedom. But what about the last type of type freedom - self-instantiation?

(6) In Quality and Concept my methodological idea was to separate issues of the logical paradoxes from issues in intensional logic as such - that is, to separate issues of the paradoxes from issues of the logic for linguistic contexts in which standard substitutivity principles do not hold. The way to do this is to construct a first-order theory for intensional abstraction and then, at a later stage, to single out a distinguished logical constant for the predication relation and to construct a theory for it. This way the logical paradoxes and their resolution can be confined to this later stage.

How are the paradoxes to be resolved ideally? Does anyone really know? I do not think so, and I think it is unrealistic to think that this situation is likely to change substantially any time soon. However, it is relatively straightforward to adapt the known approaches to the paradoxes to first-order intensional logic. As an illustration, I indicated in Quality and Concept (a) how an iterative approach could be followed and (b) by invoking the idea of context-relativity (which Charles Parsons and Tyler Burge favor), a resolution of the liar paradox could be incorporated. But this was only an illustration, as I made clear in the book. I also stated that a "fixed-point" approach like that of Gilmore and Feferman would also be attractive and easy to adapt to our first- 
order intensional logic. I am very pleased now to see that this general idea has been studied in depth by a number of people - Bill Reinhardt, Peter Aczel, Raymond Turner, Gennaro Chierchia, and others. Some people have mistakeningly thought that in the book I was advocating the iterative approach, which standardly prohibits self-instantiation, over approaches (like fixed-point approaches or Michael Jubien's approach) that allow self-instantiation. But I explicitly stated otherwise. The reason that I did not pursue either the iterative approach or a fixed-point apprbach in greater detail is that I believe, and continue to believe, that, although they are extremely elegant and very useful theoretically, they still do not yield a full understanding and resolution of these pathologies. For all we know, this theoretical ideal lies beyond our abilities. For this reason, the two-stage methodology I have urged seems unassailable. I can see nothing short of a full ideal resolution of the paradoxes that could justify anyone in departing from it.

A special advantage of this two-stage methodology is that it permits one to construct complete theories of PRPs at the first stage, i.e., complete logics for first-order languages with identity and intensional abstraction. In Quality and Concept I showed that such first-order intensional logics are complete relative to certain technical notions of validity that are defined by means of the intensional algebraic semantic method, and I advanced the philosophical thesis that these technical notions of validity are in fact the standard notions of validity (or at least that they resemble the standard notions in all respects relevant to genuine completeness results). From the technical result and the philosophical thesis it follows that first-order intensional logic is genuinely complete.

This argument is parallel to that used to show that elementary firstorder logic with identity is genuinely complete: the logic is proved complete relative to a certain defined notion of validity, and this technical result is then combined with the philosophical thesis that this defined notion is the same as (or resembles in philosophically relevant respects) the standard notion. In the case of elementary first-order logic with identity, the philosophical thesis has been subjected to much critical scrutiny, and something like a consensus has emerged in support of it. In the case of first-order intensional logic, the philosophical thesis strikes many people as highly intuitive. Nevertheless, some commentators (for example, Nino Cocchiarella and I. G. McFetridge) have expressed doubts. ${ }^{3}$ According to such doubts, the technical complete- ness result might be a mere artifact of a mistakenly narrow definition of validity that results from using an overly liberal definition of a model (in much the same way that Henkin's quasi-completeness result for higher-order extensional logic is a consequence of the liberal notion of a general model). Specifically, if certain appropriate auxiliary closure conditions were imposed on the models, perhaps completeness would no longer follow; perhaps incompleteness could be derived. So go the doubts. However, these doubts are unfounded as I will now explain.

In intensional algebraic semantics, a model consists of $D, P, K, G$, Id, Nec, $T$, conj, neg, exist, pred ${ }_{k}, \mathbf{I}$. $D$ is an infinite domain. $P$ is a prelinear ordering on $D$ that induces a partition of $D$ into subdomains $D_{-1}, D_{0}, D_{1}, D_{2}, \ldots$ (thought of as being comprised, respectively, of nonPRPs, propositions, properties, binary relations, ternary relations, etc.). $K$ is a class of functions on $D$ telling us the alternate (or possible) extensions that the elements of $D$ have. (For example, for each extension function $H$ in $K$ and each property $u$ in $D_{1}, H(u)$ is a subset of $D$.) $G$ is a distinguished function in $K$ that tells us the actual extensions of elements of $D$. Id is a distinguished element of $D_{2}$ to be thought of as the identity relation; accordingly, for all $H$ in $K, H(\mathrm{Id})=\{x y \in D: x=y\}$. Nec is a distinguished element of $D_{1}$ to be thought of as the property of necessity; accordingly, for all $H$ in $K, H(\mathrm{Nec})=\left\{x \in D_{0}: H^{\prime}(x)=T\right.$ for all $\left.H^{\prime} \in K\right\} . T$ is a class of transformations, which are functions that take relations to, say, their converses, reflexivizations, expansions, and so forth. The remaining items are basic logical operations for conjunction, negation, existential generalization, and predication. (For example,

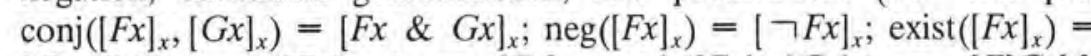
$[(\exists x) F x] ; \quad \operatorname{pred}_{0}\left([F x]_{x}, v\right)=[F v] ; \operatorname{pred}_{0}\left([F x]_{x},[G y]_{y}\right)=\left[F[G y]_{y}\right]_{,}$ $\operatorname{pred}_{1}\left([F x]_{x},[G y]_{y}\right)=[F[G y]]_{y} ; \operatorname{pred}_{2}\left([F x]_{x},[R y z]_{y z}\right)=[F[R y z]]_{y z} ;$ etc. Finally, $\mathbf{I}$ is an interpretation of our first-order intensional language. Specifically, I a function that assigns to variables elements of $D$ and that assigns to $n$-ary predicates elements of $D_{n}$; moreover, $\mathbf{I}\left({ }^{\prime}=\right.$ ') is Id and $\mathbf{I}\left({ }^{\prime} N\right.$ ') $=$ Nec. (' $N$ ' is a distinguished 1 -ary predicate for necessity. If other distinguished logical properties and relations besides Nec and Ic were adjoined to our models, corresponding distinguished logical predicates could then be adjoined to the language.) Denotation and truth in a model may then be defined inductively. In turn, validity may be defined as truth in all models. We can show that, relative to this notion of validity, the valid formulas of our first-order intensional language have a complete axiomatization. 
Now consider two strong closure conditions on models (described by Cocchiarella and McFetridge, respectively). First, the set $K$ of alternate extension functions must always be maximal, that is, it should not be possible to add further extension functions $H$ to $K$ without contradicting one of the original conditions in the definition of model. Second, for every subset $s$ of $D$ and every extension function $H$ in $K$, there must be an item $x$ in the subdomain $D_{1}$ of properties such that $H(x)=s$. (Notice that, if a model satisfies this closure condition, $D$ must be a proper class. For the closure condition implies that there are as many properties in $D$ as there are subsets of $D$. So if $D$ were a set, the closure condition would contradict Cantor's power-set theorem.)

THEOREM. First-order intensional logic is complete even if the strong closure conditions are imposed on models.

In broad outline the proof goes as follows. We follow the Henkin-style proof given in Quality and Concept except that a proper class of individual constants are adjoined to the language, and for all distinct individual constants $c$ and $d$, the sentence $c \neq d$ is adjoined to the theory. In the Henkin model that results, these individual constants will comprise the subdomain of individuals. To obtain a model meeting the second closure condition, we massage this model in appropriate ways. First, partition this subdomain into denumerably many proper classes $d_{-1}, d_{0}, d_{1}, d_{2}, \ldots$ The first of these proper classes $d_{-1}$ will be the subdomain of individuals in our new model. Then the $i$-th proper classes $(i \geqslant 0)$ will be adjoined to the old subdomain $D_{i}$ to form the new subdomain of $i$-ary intensions, and the functions $H$ in $K$ and the fundamental logical operations will be adjusted accordingly. When done properly the result is a model of the theory that meets the second closure condition. What makes this construction possible is the fact that our models have a single, unified domain $D$ in which individuals, propositions, properties, and relations are taken as primitive entities. Finally, concerning the first closure condition, it is straightforward to show that every $K$-maximal extension of the new model is also a model of the theory. The key to the proof is the fact that, for any algebraic intensional model, the values of all identity and necessity sentences must be the same in a $K$-extension of a model as they are in the model itself. $^{4}$

This and similar results provide strong evidence that doubts about the genuine completeness of first-order intensional logic are unfounded and that the two-stage methodology (according to which intensional logic is treated prior to treating the logic for the predication relation) is vindicated. $^{5}$

\section{HOW TO DEAL WITH TYPE FREEDOM IN SEMANTICS}

We have seen that a common way of justifying an ontology of PRPs is to show that this ontology is called for in semantics: given that PRPs are what predicates and sentences mean and given that PRPs are what intensional abstracts denote, a theory characterizing the semantical features of a language must make ontological commitment to PRPs. But how extensive must this commitment be? The answer is that it can be relatively weak, as I will show.

My remarks are organized around the question of how a semantical theory should deal with the various types of type freedom. The answer to this question depends on one's goals. Suppose that one's goal is to state what the expressions in an arbitrary language mean. If the constants of the language have synonyms in the language of one's PRP theory (or if, as a limiting case, the language is the language of the PRP theory), we can accomplish the goal by an axiomatic theory. Such a theory would have the following schema for (closed) sentences ' $A$ ' and open-sentences ' $B u_{1} \ldots u_{n}$ ':

$$
\begin{aligned}
& \text { ' } A \text { ' means }[A] \text {. } \\
& \text { ' } B u_{1} \ldots u_{n} \text { ' means }\left[B u_{1} \ldots u_{n}\right]_{u_{1} \ldots u_{n}} \text { ' }
\end{aligned}
$$

where 'means' is taken as a primitive constant. For example, 'Something runs' means (expresses) the proposition that something runs, and ' $u_{1}$ runs' means (expresses) the property of being a $u_{1}$ such that $u_{1}$ runs. All that is needed is a background PRP theory that is fitted out with intensional abstraction. An advantage of this approach is that PRP semanticists need not take any stand on the question of self-constituency and self-instantiation, which are the two most controversial types of type freedom.

Next suppose that one wishes to specify (perhaps in an indefinite way) what the expressions in an arbitrary language mean, where the primitive vocabulary of the language exceeds that of the language of our background PRP theory. For example, if ' $F u$ ' expresses the property $x$ and ' $G u$ ' expresses the property $y$, what do ' $F u$ \& $G u$ ', ' $\neg F u$ ', and 
' $F[G u]_{u}$ ' mean? To do this kind of general semantics, I see no alternative but to adopt a PRP theory that posits fundamental logical operations such as conjunction, negation, singular prediction, and so forth The most straightforward way to formulate such a theory is to adjoin to one's background PRP theory distinguished logical predicates for these operations ('Conj ${ }^{3}$, ' $\mathrm{Neg}^{2}$ ', 'Pred ${ }^{3}$,', and so forth). For example, 'Fu \& $G u$ ' would mean the property $z$ such that $\mathrm{Conj}^{3} x y z$; ' $\neg F u$ ' would mean the property $z$ such that $\mathrm{Neg}^{2} x z$; and ' $F[G u]_{u}$ ' would mean the proposition $z$ such that Pred $^{3} x y z$. An advantage of this approach is that, as before, PRP semanticists' need not take a stand on whether there is any self-constituency or self-instantiation. If such phenomena truly exist, they will automatically be accommodated by this approach; no special logical apparatus or associated principles would be needed. ${ }^{6}$

Now a property theory with a primitive intensional abstraction operation and distinguished logical predicates for identity and the predication relation (but no distinguished logical predicates for the fundamental logical operations) might also be able to do this kind of semantics just discussed. In light of new work [such as that of Gennaro Chierchia and Raymond Turner (1987) and Turner (1987)], this approach appears promising. There are, however, two potential worries. First, it would require PRP semanticists to take intensional abstraction as a primitive (rather than defined) operation. This in turn would require us to accept an ultimate bifurcation of logic into extensional and intensional (rather than to explain prima facie intensional constructions as surface phenomena definable within a logical setting that is thoroughly extensional).? Second, this approach requires PRP semanticists to take a stand on how ideally to resolve the paradoxes involving the predication relation. But for the purposes at hand nothing forces PRP semanticists to take on this heavy challenge. Therefore, it would be methodologically much wiser to defer this challenge. In view of these two drawbacks, it is hard to see why we should not adopt the neutral and secure semantical method sketched earlier.

Suppose one wished to define (by an inductive-turned-direct definition) the constant 'mean' as it occurs in the style of semantical theory sketched above, including as a limiting case a semantical theory for the very language in which the semantical theory is stated. This can be done using no additional machinery beyond that of a standard well-founded set theory. No commitment to self-constituency or self-instantiation must be made. The reason standard well-founded set theory suffices is that there is no reason in the semantic theory to assert any special relationships between PRPs and sets (for example, that for every set, there are infinitely many properties whose instances are exactly the elements of that set). As far as the set theory is concerned, the PRPs collected in a set are on a par with particulars or any other primitive urelements. As a result, the sets needed to validate an inductive-turneddirect definition of the meaning relation over the field of linguistic expressions and PRPs can be standard well-founded sets.

Semanticists can thus do a considerable amount of semantics without even using a distinguished logical predicate for the predication relation. Consequently, the question of self-instantiation does not even come up at this stage of semantical theorizing. In semantics, it becomes necessary to use a constant for the predication relation only when one wishes to supplement one of the above theories of meaning with a theory of truth conditions. To obtain such a theory, we adopt the following definitions:

A predicate or open sentence is true of an item if and only if the item is an instance of the property that is the meaning of the predicate or open sentence.

and

A sentence is true if and only if the proposition that is the meaning of the sentence is a true proposition.

Here, and not before, does it become necessary for semanticists to use a constant for the predication (instantiation) relation. Once this is available, one can define truth for propositions. However, truth for propositions can be defined without explicitly supposing that any properties are instances of themselves. Instead, the following sort of definition suffices for this purpose:

$[A]$ is true if and only if $(\exists u) u$ is an instance of $[A]_{u}$.

(Or more generally, $x$ is true $\operatorname{iff}_{\mathrm{dr}}(\exists u) u$ is an instance of $\exp (x)$. The operation exp is the distinguished logical operation that adds on an extra degree to a PRP; for example, $\exp ([F a])=[F a]_{u}$ and $\exp \left([F u]_{u}\right)=$ $\left.[F u]_{u v}\right)$. Concerning questions of self-constituency and self-instantiation, semanticists may, as before, remain neutral. These are independent questions in logical theory (or metaphysics) that do not arise when one is attempting simply to specify the conditions under which an expression is true of an object or the conditions under which a sentence is 
true. It is just not the business of semantics to say what expressions are true of what objects. For example, it is not the business of semantics to say whether the term 'spy' is true of a certain suspect; by the same token, it is not the business of semantics to say whether, say, the term 'self-identical' is true of a certain metaphysically and epistemologically interesting suspect, namely, the very property of self-identity. The job of semantic theory here is at most to say under what conditions the expression would be true of the object. Similarly, it is not the job of semantics to say whether a sentence is true. For example, it is not the job of semantics to say whether ' $(\exists x) x=$ the most dangerous spy' is true. By the same token, it is not the job of semantics to say whether the self-constituency sentence ' $(\exists x) x=[x$ is not true $]$ ' is true. The job of semantics here is at most to say under what conditions the sentence would be true. As I have indicated, these semantical tasks can be achieved without making commitment to self-constituency or to selfinstantiation.

So must semantics ever make a commitment to self-constituency or to self-instantiation? I submit that semanticists as semanticists can always remain neutral about whether there is or is not self-constituency. There are no issues in semantics (i.e., the theory of fundamental wordworld relations) that force one to take a stand here. The question of whether there is self-constituency belongs to logic (or metaphysics). If self-constituency should happen to exist, the logical apparatus suggested in the above PRP semantic methods would automatically accommodate it. No special steps need to be taken by semanticists as semanticists. (Later on, I will examine the philosophical arguments for and against self-constituency.)

The situation with self-instantiation is somewhat similar. In virtually all intellectual settings, semanticists can remain neutral on the issue of self-instantiation. However, there is a special intellectual setting in which they might not. Namely, a setting in which, for reasons independent of semantic theory, they hold that it is illegitimate to use set theory for any purpose. For example, advocates of certain no-class theories hold this because they think that there really are no such things as sets, that set theory is a mere artifice resulting from an erroneous conflation of certain natural notions such as property and ordinary aggregation. (See chapter 5 "Class" in Quality and Concept for a discussion of this thesis.) If a semanticist holds this position, then and only then might there be a need to posit self-instantiated properties.
And in this case, the need would arise only when one seeks to give a definition (inductive-turned-direct) of the meaning relation for the language of one's own semantic theory (or some language of equivalent expressive strength). If in this setting one does not have available any set theory for stating the definition, one must turn to a property theory for the machinery for turning the inductive definition into a direct definition. It is just here - and at no other place - that self-instantiation is needed in semantics. Self-instantiation is unavoidable here, for the definition would be incorrect unless the pair ' 'means', meaning is an instance of the meaning relation. And this is a case of self-instantiation. The conclusion is that in semantics one can get on without positing self-instantiation unless one holds, for reasons independent of semantics, that sets are not real. And even then, one need not posit self-instantiation at any point prior to giving a definition of the meaning relation for the language of one's own PRP theory (or a language of equivalent strength). If you do not hold that sets are not real, you will need to look beyond semantics proper for a reason to posit self-instantiation. (At the close of my remarks, I will indicate where you might look for such a reason.)

On the view I have been advocating, semantics is a relatively weak discipline that can remain neutral on a great many issues that at bottom belong to logic or some other discipline outside linguistics. This view clashes with a more inflated conception of semantics that is fashionable in some contemporary circles. The inflated conception is, in my opinion, based on confusions about the proper boundaries between semantics and other disciplines (especially logic), confusions that blur the proper hierarchical organization of these disciplines. Logic is the theory of valid reasoning. On my view, the best development of logic is (or at least includes) a theory of PRPs. In this theory, validity (and also entailment and analyticity) is characterized in terms of various auxiliary notions - identity, contrariety, predication, truth, necessity, and so forth. These notions are purely logical, not linguistic. Semantics, by contrast, is the theory of the fundamental relations between words and the world. The most fundamental word/world relations are meaning and referring. (We need not take a stand here on the question of whether referring is a species of meaning or a relation derivative from meaning or a primitive relation in its own right.) Given the above purely logical notions and given the primary semantical notion of meaning, a variety of derivative semantical notions are definable: synonyms 
$\left({ }_{\mathrm{df}}\right.$ expressions whose meanings are identical), antonyms $\left(\boldsymbol{~}_{\mathrm{df}}\right.$ expressions whose meanings are contraries), a predicate true of an item $\left(=_{\mathrm{df}}\right.$ an expression whose meaning has the item as an instance), true sentence $\left({ }_{\mathrm{df}}\right.$ a sentence whose meaning is a true proposition), necessary sentence $\left({ }_{\mathrm{df}}\right.$ a sentence whose meaning is a necessary proposition), valid sentence ( ${ }_{\mathrm{df}}$ a sentence whose meaning is a valid proposition), and so forth. Accordingly, specific claims about these derivative matters - synonyms, antonyms, predicates true of items, true sentences, necessary sentences, valid s'entences, and so forth - result from a combining (1) these definitions, (2) elementary semantical facts about which PRPs are the meanings of which linguistic expressions, and (3) languageindependent facts about which PRPs are identical, contrary, instantiated, true, necessary, valid, and so forth. Semanticists have finished their job as semanticists when they have completed (1) and (2); issues concerning (3) are not the province of semantics as such. In view of this, semanticists can and should remain neutral on a great many questions concerning (3), questions that properly belong to other disciplines. Such questions can be answered independently of linguistics, and once they are answered, the answers to the derivative semantical questions follow immediately and trivially, providing no new theoretical illumination.

I am inclined to much the same view of thematic-role semantics. The elaborate structures posited there do not belong to semantics as such; they belong to epistemologically antecedent, more general a priori disciplines, namely, logic and metaphysics. The job of the lexical semanticist is merely to tell us what various lexical items mean. This can be done homophonically if the semantic metalanguage includes the object language. The rest of the work is done automatically by general logical and metaphysical principles that are wholly languageindependent. I have yet to see any linguistic examples that require abandoning this simple traditional epistemological picture of the hierarchy of disciplines.

\section{AGAINST IDENTIFYING PROPERTIES AND PROPOSITIONAL FUNCTIONS}

Formalizations of PRP theory run into some technical complexities in connection with the treatment of free and bound terms occurring in PRP-abstracts. ${ }^{8}$ Many of these complexities can be avoided by adopting the thesis that properties and propositional functions are identical. ${ }^{9}$ The purpose of this section is to present some significant reasons not to adopt this thesis despite its short-term technical advantages.

Philosophically, the propositional-function thesis seems unacceptable on at least two counts. First, it is highly counterintuitive. How implausible that familiar sensible properties are functions - the color of this ink, the aroma of coffee, the shape of your hand, the special painfulness of a burn or itchiness of a mosquito bite. No function is a color, a smell, a shape, or a feeling. Or consider the fact that navy blue is darker than yellow and that it covers the surface of my pen. No function is darker than another function, and no function covers the surface of my pen. To assert otherwise seems to be an old-fashioned category mistake. ${ }^{10}$

In addition to intuitive objections to the propositional-function thesis, a second philosophical objection is that the thesis threatens the prospect of certain explanations in epistemology, phenomenology, and philosophical psychology. For example, how are we to explain what is going on in the interplay of sensation and cognition when a person sees that two objects have some sensible quality in common? Or how are we to explain why various shades of color can look so similar? At best, the identification of properties and propositional functions complicates our epistemology, phenomenology, and philosophical psychology in connection with these and kindred phenomena.

My aim, however, is not to dwell on such philosophical objections to the propositional-function thesis, serious as they are. Rather, my aim is to discuss four logical difficulties facing the thesis.

(1) The first problem derives from the fact that functions are usually treated extensionally. That is, the following extensionality principle holds for all functions $f$ and $g:(\forall x)(f(x)=g(x)) \rightarrow f=g$. But this is wrong. There always exist functions $f$ and $g$ such that, even though $f$ and $g$ yield the same values for the same actual arguments, they could yield different values for some possible arguments. That is, even though $(\forall x)(f(x)=g(x))$, nevertheless $\diamond(\exists x)(f(x) \neq g(x))$. In this case, $f \neq g$. Hence, a counterexample to the extensionality principle. This problem could be overcome treating functions intensionally. To do this, one would reject the original extensionality principle; in its place, one might adopt a modal extensionality principle: $\square(\forall x)(f(x)=g(x) \rightarrow f=g$. This move, however, does complicate things.

(2) The second problem is far more serious. One of the main purposes of PRP theory is to provide a logical framework for treating the 
propositional attitudes - belief, decision, memory, and so forth. It is now widely recognized that the sort of propositions suited to serve as objects of the attitudes must be very fine-grained. ${ }^{11}$ For example, if two properties, say, being an $x$ such that $x F \mathrm{~s} \neq$ being an $x$ such that $x G$ s, then for every $x$, the proposition that $x F \mathrm{~s} \neq$ the proposition that $x G \mathrm{~s}$. The intuitive idea here is that, if these two properties differ in any respect, then it will always be possible in principle for some person to have some propositional attitude to the proposition that $x F_{\mathrm{S}}$ and to fail to have that attitude toward the proposition that $x G$ s. Differences between properties are always reflected in differences between propositions formed from those properties. The logico-linguistic evidence supporting this principle of the distinctiveness among propositions is overwhelming.

This principle of distinctness among propositions entails (by contraposition and quantifier interchange) the following fine-grained principle of identity for properties: if, for some $x$, the proposition that $x F_{\mathrm{S}}=$ the proposition that $x G \mathrm{~s}$, then being an $x$ such that $x F \mathrm{~s}=$ being an $x$ such that $x G$ s. This and kindred principles of property identity are formalized in my first-order PRP theory T2. ${ }^{12}$ These property-identity principles comprise pretty much the object-language statement of the fine-grained conception of synonymy that Alonzo Church calls synonymous isomorphism and that he believes is required for treating the propositional attitudes. Church arrived at his conception by tightening up Carnap's principle of intensional isomorphism in response to various counterexamples. ${ }^{13}$ Church has tried with very mixed success to formalize his conception in a higher-order ramified intensional logic called Alternative $(0) \cdot{ }^{14}$

To show that it is a mistake to identify properties and propositional functions, we will make use of two intuitively compelling syntactic principles. First, if the word 'that' and a noun phrase $\alpha$ and a primitive verb phrase (i.e., a primitive predicate) ' $F$ s' are concatenated, the result is a well-formed 'that'-clause 'that $\alpha$ Fs'. Second, if ' $x$ ' is a variable and ' $F$ s' is a primitive verb phrase 'being an $x$ such that $x F \mathrm{~s}$ ' is a wellformed gerund. And we use the following semantical principles:

(A) Any property can in principle be expressed by a primitive verb phrase ' $F$ s'.

(B) If ' $F$ ' is a primitive verb phrase, the gerund 'being an $x$ such that $x F$ ' denotes the property expressed by ' $F$ s'.
(C) If the variable ' $x$ ' is assigned the individual $x$ as its value and the primitive verb phrase ' $F$ s' expresses the property of being an $x$ such that $x F \mathrm{~s}$, then the 'that'-clause 'that $x F \mathrm{~s}$ ' denotes the proposition that $x F \mathrm{~s} .^{15}$

(D) If ' $F$ s' and ' $G$ s' are primitives verb phrases, then the following holds:

If, for some $x$, that $x F_{\mathrm{S}}=$ that $x G \mathrm{~s}$, then being an $x$ such that $x F \mathrm{~s}=$ being an $x$ such that $x G$ s.

(E) If a variable ' $x$ ' is assigned the individual $x$ as its value and a primitive verb phrase ' $F$ s' expresses a propositional function $f$, then the 'that'-clause 'that $x F$ s' denotes the proposition that is the value of $f$ applied to argument $x$, that is, $f(x)$.

(A)-(C) are intuitively compelling principles of informal semantics. It would seem unreasonable to abandon these basic principles just to save the propositional-function thesis. (D) presents the fine-grained principle of property identity discussed above. (E) is a fundamental principle of all standard propositional-function semantic theories. (E) is a conditional whose antecedent implies that the primitive verb phrase ' $F$ s' express a propositional function $f$. However, given that the primitive verb phrase ' $F$ s' expresses a property, the antecedent of $(E)$ implies that the propositional function $f$ and this property are identical. If, as I maintain, this is false, then the entire conditional $(E)$ is true. So we are free to use (E) in our argument against the propositional-function thesis. With principles $(\mathrm{A})-(\mathrm{E})$ in place, we can now disprove the propositional-function thesis. Here is the proof.

Proof. Let $g$ be a constant propositional function characterized by the equation: $(\forall u)(g(u)=$ the proposition that $x$ flies), where $x$ is some arbitrarily chosen item, say, the number nine. Then by the propositionalfunction thesis, it follows that $g$ is a property. So by (A), a primitive verb phrase ' $G$ s' could express $g$. Let the variable ' $x$ ' be assigned $x$ (i.e., the number nine) as its value. Then, by (E), the 'that'-clause 'that $x G \mathrm{~s}$ ' denotes the proposition that $x$ flies. By $(\mathrm{C})$, the 'that'-clause 'that $x$ flies' denotes the proposition that $x$ flies. So 'That $x G \mathrm{~s}=$ that $x$ flies' is true on the above assignment. Therefore, the sentence 'For some $x$, that $x G \mathrm{~s}=$ that $x$ flies' is true. Thus, by (D), 'being an $x$ such that $x G \mathrm{~s}=$ being an $x$ such that $x$ flies' is true. It follows that these two gerunds 
denote the same property. Consequently, by (B), the primitive verb phrases ' $G$ s' and 'flies' express the same property. Given the propositional-function thesis, this property is really a propositional function. But the propositional function expressed by ' $G$ s' is $g$, so it follows that 'flies' expresses $g$. Now select some individual $y$ that actually flies, say, this hummingbird, and let the variable ' $y$ ' be assigned $y$ as its value. Given that the primitive verb phrase 'flies' expresses the propositional function $g$, it follows by (E) that the 'that'-clause 'that $y$ flies' denotes the proposition that' is the value of $g$ applied to the argument $y$, that is, $g(y)$. However, by $g$ 's characterizing equation, $g(y)=$ the proposition that $x$ flies (where $x=$ the number nine). Thus, the 'that'-clause 'that $y$ flies' denotes the proposition that $x$ flies (where $x=$ the number nine). However, by (B), 'that $y$ flies' also denotes the proposition that $y$ flies (where $y=$ this hummingbird). It follows that the proposition that $y$ flies $=$ the proposition that $x$ flies (where $y=$ this hummingbird $\neq 9=x$ ). But this is absurd. The proposition that $y$ flies is true; the proposition that $x$-flies is false. (This hummingbird flies; the number nine does not.) Since the propositional-function thesis leads to this absurdity, it follows that it is false. ${ }^{16}$

Two observations are in order. First, this argument does not show that propositional functions cannot be used in constructing models for intensional logic. In particular, one could construct artificial models such that every proposition in the model is the value of at most one propositional function in the model. In models like this the above sort of problem would not occur. Such models could be used for a variety of specific logical tasks. However, there is still a problem. None of these artificial models is a natural model of propositional functions, for in a natural model there will always be more than one - indeed, there will always be infinitely many - propositional functions having the same proposition as one of the values. [For example, in a natural model containing the natural numbers there will be infinitely many propositional functions $g_{i}, i \geqslant 1$, that have as a value the proposition that $0=1$; functions like this might be characterized thus: $(\forall x)$ (if $x$ is a natural number less than $i$, then $g_{i}(x)=$ the proposition that $0=1$; otherwise, $g_{i}(x)=$ the proposition that $x=x$ ). J But a condition of adequacy on any general semantical method is that the models it provides should include the natural model(s) for the entities it is supposed to model. Therefore, a general semantical method based on the above artificial models cannot be adequate.
The second observation concerns a weakening of the propositionalfunction thesis. We know that, for every proposition, there are infinitely many propositional functions having that proposition as a value. But given $(A)-(E)$, we have shown that at most one of these propositional functions could possibly be a property. So it appears that a propositionalfunction theorist has no choice but to weaken the original propositionalfunction thesis in the following way. Originally, the thesis was that all and only properties are (unary) propositional functions. According to the weakened thesis, all properties would still be (unary) propositional functions; however, the converse would not hold. On the contrary, most (unary) propositional functions would not be properties. [On this weakened thesis, the propositional function $f$ having the following defining equation might be an example of a propositional function that is really a property (i.e., the property of flying): $\square(\forall x)(f(x)=$ the proposition that $x$ flies). By contrast, the propositional functions $g_{i}$ characterized above would not be genuine properties according to the weakened thesis.] True enough, this weakened propositional-function thesis avoids the difficulty given above. However, it does so at the price of making a mystery of the distinction between propositional functions that are supposed to be properties and those that are not. What is it about the propositional functions that are supposed to be properties that makes them special? The answer presumably is that these propositional functions are somehow more "natural" than others. The problem is that there evidently is no way to spell out clearly and precisely what this means without implicitly or explicitly using the logically prior idea of what it is to be a property (or related ideas not belonging to propositional-function theory as such). But if this is so, it would appear that despite its technical appeal, the weakened propositional-function thesis inevitably leaves something out; it masks the true logical structure of the subject. Conceptually, the right course is therefore to develop a theory of properties directly. Only such a theory can lay bare the true logical structure of PRPs.

(3) The third problem I wish to raise confronts even the weakened thesis that all (but not only) properties are unary propositional functions. As with the second problem, the third problem arises in the context of the logic for the propositional attitudes, which demands that very fine-grained distinctions be made among propositions. Let us introduce two new primitive verb phrases (primitive predicates) 'rajneeshes' and 'fondalees' - by stipulating that: 
(1) Being an $x$ such that $x$ rajneeshes $=$ being an $x$ such that $x$ follows Rajneesh.

(2) Being an $x$ such that $x$ fondalees $=$ being an $x$ such that Jane Fonda follows $x .^{17}$

(Jane Fonda seems to follow many people as time goes on; maybe it will be Rajneesh next.) According to the propositional-function thesis, the following identities hold:

(3) $\quad(\lambda x)(x$ rajheeshes $)=$ being an $x$ such that $x$ rajneeshes.

(4) $\quad(\lambda x)(x$ follows Rajneesh $)=$ being an $x$ such that $x$ follows Rajneesh.

(5) $\quad(\lambda y)(y$ fondalees $)=$ being a $y$ such that $y$ fondalees.

(6) $(\lambda y)$ (Jane Fonda follows $y)=$ being a $y$ such that Jane Fonda follows $y$.

From (1), (3), and (4) it follows that:

$$
(\lambda x)(x \text { rajneeshes })=(\lambda x)(x \text { follows Rajneesh }) .
$$

Apply each side of this identity to the argument Jane Fonda. The result is:

\section{$(\lambda x)(x$ rajneeshes $)(J a n e$ Fonda)}

$$
=(\lambda x)(x \text { follows Rajneesh }) \text { (Jane Fonda). }
$$

Then by $(\mathrm{C})$ and $(\mathrm{E})$ we have:

(7) The proposition that Jane Fonda rajneeshes $=$ the proposition that Jane Fonda follows Rajneesh.

Similarly, from (2), (5), and (6), it follows that:

$$
(\lambda y)(y \text { fondalees })=(\lambda y)(\text { Jane Fonda follows } y) .
$$

Apply each side of this identity to the argument Rajneesh. The result is:

$$
\begin{aligned}
& (\lambda y)(y \text { fondalees }) \text { (Rajneesh) } \\
& =(\lambda y)(\text { Jane Fonda follows } y) \text { (Rajneesh). }
\end{aligned}
$$

Then by $(\mathrm{C})$ and $(\mathrm{E})$, we have:

(8) The proposition that Rajneesh fondalees $=$ the proposition that Jane Fonda follows Rajneesh.
From (7) and (8) it follows that:

The proposition that Jane Fonda rajneeshes $=$ the proposition that Rajneesh fondalees.

But this seems wrong. When a person consciously and explicitly thinks that Jane Fonda rajneeshes must that person be consciously and explicitly thinking that Rajneesh fondalees? It certainly does not seem so. ${ }^{18}$ Thus, the weakened propositional-function thesis seems mistaken.

We have isolated another prima facie difficulty in the propositionalfunction thesis. It would be desirable to have a diagnosis of what has gone wrong. I will venture one, but I should emphasize that the problem should not be confused with the diagnosis (or with the technical apparatus used to state the diagnosis). Independently of the diagnosis, we have established that the propositional-function thesis leads to a prima facie problem.

According to the diagnosis, the objects of the propositional attitudes are so fine-grained that in the case of relational propositions the order in which relations are predicated of arguments is reflected in the identity of the propositions that are the outcome. For example, the relational property rajneeshing results from predicating the binary relation of following of Rajneesh, and in turn the relational proposition that Jane Fonda rajneeshes results from predicating this property of Jane Fonda. On the other hand, the relational property fondaleeing results form predicating the inverse of the binary relation of following of Jane Fonda, and in turn the relational proposition that Rajneesh fondalees results from predicating this property of Rajneesh. In symbols,

$$
[R j]=\operatorname{pred}\left(\operatorname{pred}\left([F x y]_{x y}, r\right), j\right) \neq \operatorname{pred}\left(\operatorname{pred}\left([F x y]_{y x}, j\right), r\right)=[F r] .
$$

The reason that the propositional-function approach does not mark the distinction between these two propositions is that the order in which the corresponding propositional functions are applied to the argument is not analogously reflected in the identity of the outcome:

$$
(\lambda x)(R x)(j)=(\lambda x y)(F x y)(j)(r)=(\lambda y x)(F x y)(r)(j)=(\lambda y)(F y)(r) .
$$

Ironically, this and kindred phenomena are exactly the ones that make a propositional-function approach technically simpler than an approach that takes properties and relations as primitive entities not reducible to propositional functions. What the above example seems to show is that 
this very simplification blurs genuine distinctions among the type of propositions that figure in the logic for the propositional attitudes. ${ }^{19}$

I can think of two ways in which propositional-function theorists might try to regain the missing distinctions. First, they might try to regain them by introducing into their object theory a special primitive predicate for the application of a propositional function to an argument. For example, they might introduce the binary predicate 'App', where 'App $(u, v)$ ' is intended to mean the following:

The result of applying propositional function $u$ to argument $v$ is a true proposition.

Then, if these propositional-function theorists are willing to give up principle (E), they could claim:

(9) That $R j=$ that $\operatorname{App}((\lambda x)(F x r), j)$.

and

$$
\text { (10) That } F r=\text { that } \operatorname{App}((\lambda y)(F j y), r) \text {. }
$$

Because:

$$
\text { That } \operatorname{App}((\lambda x)(F x r), j) \neq \text { that } \operatorname{App}((\lambda y)(F j y), r) .
$$

the missing distinction:

$$
\text { That } R j \neq \text { that } F r \text {. }
$$

would be regained. This is to say:

That Jane Fonda rajneeshes $\neq$ that Rajneesh fondalees.

However, this way out of the problem is not acceptable. Not only does it involve giving up principle (E), which is the central principle of standard propositional-function semantic theories, but also it is manifestly mistaken on its face. For (9) and (10) are plainly false: a person could consciously and explicitly think that Jane Fonda rajneeshes without consciously and explicitly thinking that the result of applying the propositional function $(\lambda y)(F x r)$ to Jane Fonda is true. Indeed, someone could think the former proposition and not even have the concept of applying a function to an argument! So this way of trying to solve the problem must be abandoned.

The second way in which propositional-function theorists might try to regain the missing distinctions is by holding that these distinctions are pragmatic not semantic. That is, they could hold that strictly and literally:

The proposition that Jane Fonda rajneeshes $=$ the proposition that Rajneesh fondalees.

is true. But in conversation when you say:

I think that Jane Fonda rajneeshes.

what you would mean differs from what you would mean when you say:

I think that Rajneesh fondalees.

This pragmatic difference can then be explained by means of Gricean rules of conversation without calling into question the above strict and literal identity. So goes the pragmatic solution.

However, this kind of pragmatic solution creates a special problem for propositional-function theorists. In Section 5, I will show that such pragmatic solutions require an independent solution to the paradox of analysis. Specifically, such pragmatic solutions require positing a distinction between analyzed intensions and unanalyzed intensions. That is, there must be two types of intension, analyzed and unanalyzed. Given this, propositional-function theorists who advocate the pragmatic solution are faced with two grave problems. First, they are forced to decide which type of 0 -ary intension - analyzed or unanalyzed - are to be the values of propositional functions. Inevitably, the choice will be utterly arbitrary. Second, they must work out a theory of the other type of 0 -ary intension (i.e., the type of 0 -ary intension not chosen to be values of propositional functions). Presumably, some further logical machinery besides that provided by the propositional-function theory will be needed for this purpose, and the use of this further logical machinery will lead propositional-function theorists to a disunified general theory of PRPs. On an algebraic approach to PRPs, both of these defects - the arbitrariness and the disunity - are avoided.

(4) I have just indicated that the existence of two types of PRP analyzed and unanalyzed - creates grave problems for propositionalfunction theorists who would try to save their theory by pragmatic maneuvers. I now want to show that these grave problems are quite general: they arise as long as there is more than one type of PRP. Suppose for a moment that I am wrong about the need to introduce a distinction between analyzed and unanalyzed intensions to solve the 
paradox of analysis. No matter; there are also compelling intuitive and theoretical reasons for positing a distinction between fine-grained and coarse-grained PRPs. ${ }^{20}$ This distinction is enough to produce the same sorts of special problems for the propositional-function thesis noted in the previous paragraph. I will spell this out more fully.

Certainly both fine-grained and coarse-grained intensions exist. Consider an example. Intuitively, the thought that the glass is half empty is different from the necessarily equivalent thought that the glass is half full. (Thoughts are fine-grained 0 -ary intensions.) Not so for the conditions (situations, states of affairs) to which thoughts correspond in the world. (Conditions are coarse-grained 0 -ary intensions.) Intuitively, the glass's being half empty is the same condition (situation, state of affairs) in the world as the glass's being half full. It is just the physical condition that you are observing right there in front of you. ${ }^{16}$

Now the existence of more than one type of 0 -ary intension gives rise to the following question. If properties are treated as propositional functions, are the values of these functions thoughts, or are they conditions (situations, states of affairs)? Are the values of these functions to be identified with fine-grained or coarse-grained 0 -ary intensions? Two observations.

First, the answer seems utterly arbitrary. If properties are identified with propositional functions, what grounds are there for thinking that their values are coarse-grained rather than fine-grained or fine-grained rather than coarse-grained? No answer seems available. The propositional-function thesis requires the assumption of an arbitrary dogma on this point.

Suppose, however, that this inevitable arbitrariness is swallowed (as it should not be) and that the values of propositional functions are arbitrarily identified with one of the two types of 0 -ary intensions. How is one to develop a theory of the other type of intension? This job will require some new kind of logical machinery, machinery not used in the original propositional-function approach. My second observation is this. This new logical machinery is likely to be very much like that used in the algebraic approach to intensional entities, which is the main competitor to the propositional-function approach. If so, what is gained by not using an algebraic approach to both types of intension from the start? Furthermore, whatever the new kind logical machinery is like, it certainly must go beyond that needed by the original propositionalfunction approach. For this reason, the resulting propositional-function theory inevitably fails to provide a unified treatment of both types of intension. One type of intension will be treated one way (i.e., by means of the original propositional-function machinery); the other type will be treated some other way (i.e., by means of the additional kind of logical machinery). (Perhaps you will try to identify coarse-grained intensions with equivalence classes of necessarily equivalent fine-grained intensions. But how unintuitive and unnatural!) The algebraic approach, by contrast, provides a unified treatment of all types of intension; there is no ad hoc disanalogy in the way different types of intension are treated. ${ }^{21}$

Now let me summarize our conclusions about propositional functions. In addition to the various philosophical problems cited at the outset of this section, there seem to be four significant logical problems confronting theories that identify properties and unary propositional functions. By similar arguments, we can also show that there are four analogous problems confronting theories that identify $n$-ary relations and $n$-ary propositional functions $(n \geqslant 2)$. In view of these conclusions, we should not be tempted by the short term technical simplifications imparted by these theories. Properties cannot be reduced to unary functions (or other such entities made prominent by mathematics). Likewise, $n$-ary relations cannot be reduced to $n$-ary propositional functions. Properties and relations must be taken at face value as primitive, logically fundamental entities. By saying this, I am not suggesting that propositional functions do not exist. (Indeed, on the most economical theory of propositional functions, a unary propositional function is just a univocal ${ }^{22}$ binary relation-in-intension; a binary propositional function is just a univocal ternary relation-in-intension, and so forth.) The point is this. Once properties and relations are taken as irreducible entities, propositional functions will cease to play the pivotal role that they played in earlier formulations of intensional logic motivated by mathematics. A correct formulation of intensional logic treats properties and relations directly, and propositional functions are treated, not as something special, but as just one more kind of relation.

\section{A TRANSCENDENTAL ARGUMENT FOR FINE-GRAINEDNESS}

The are conversational contexts in which utterances of the following three sentences would be true, true, and false, respectively:
Chewing $=$ masticating.

Nobody doubts that whatever chews chews. 
(3) Nobody doubts that whatever masticates chews.

However, in most fine-grained intensional logics (e.g., my system T2), if sentences (1) and (2) are true, (3) must be true also. Hence, prima facie substitutivity paradox.

There are two reasonable responses to this problem. The first is to accept the intensional logic as literally sound and to give a pragmatic explanation of our intuitions about the divergence in truth value of utterances of (2) and (3). On this pragmatic explanation, sentences (2) and (3) would be deemed semantically equivalent. Nevertheless, utterances of (2) and (3) in an appropriate conversational context could express non-equivalent propositions. To determine exactly which propositions would be expressed conversationally, we must appeal, not just to the semantics of the language, but also to Gricean pragmatic rules of conversation. $^{23}$

The second response to the problem would be to construct a new theory that admits ultra-fine-grained intensional distinctions so that $'[(\forall x)(C x \rightarrow C x)] \neq[(\forall x)(M x \rightarrow C x)]$ ' is literally true. The idea (once suggested by Putnam $)^{24}$ is to exploit the differences in grammatical form between the two complex abstracts flanking ' $\neq$ '. Specifically, the predicate ' $C$ ' (for 'chew') is repeated in the first abstract but not in the second; so the first has the form ' $[(\forall x)(1 x \rightarrow 1 x)]$ ' whereas the second has the form ' $[(\forall x)(1 x \rightarrow 2 x)]$ '. The new theory, then, is built around the following general principle: two abstracts are codenoting only if they have exactly the same grammatical form. It turns out that such a theory is relatively easy to formulate within the general algebraic approach I have advocated. ${ }^{25}$

How are we to choose between these two responses to the puzzle the pragmatic response and the ultra-fine-grained response? The latter response is initially very appealing because it is systematic. But there is reason to doubt it. True, this response solves the above puzzle (and many similar puzzles including the original version of the famous puzzle given by Benson Mates). ${ }^{26}$ But, ironically, there are simpler versions of the above puzzle that cannot be solved no matter how fine-grained we allow PRPs to be. For example, consider any two predicates that express the same property, for example, 'chew' and 'masticate'. (Or choose some predicate ' $C$ ' and then just stipulate that a new predicate ' $M$ ' expresses the same property as that expressed by ' $C$ '.) There are conversational contexts in which someone "halfway" along in the pro- cess of mastering the predicate 'masticate' could make a true assertion by uttering, "I am sure that whatever masticates chews, but I am not sure that whatever chews masticates." In this example, the relevant two intensional abstracts have the same grammatical form: " $[(\forall x)(M x \rightarrow$ $C x)]$ ' and ' $[(\forall x)(C x \rightarrow M x)]$ ' are grammatically isomorphic. Both of these abstracts have the same grammatical form: $[(\forall x)(1 x \rightarrow 2 x)]$. Thus, the second response will not allow us to hold that these two abstracts denote distinct propositions, and, therefore, it cannot be used to solve the puzzle. Consequently, there is no choice but to invoke the first response, that is, to solve this puzzle pragmatically. (See below for details.) However, if we must resort to a pragmatic solution of this simple version of the substitutivity puzzle, uniformity demands that we use it to solve all the more complex versions, too. Moreover, if the pragmatic solution is adequate, the second response, which involves positing ultra-fine-grained intensional distinctions, would appear to be superfluous ontologically.

This conclusion raises a general methodological issue. How are we to decide which types of fine-grained distinctions to admit in intensional logic? Principles of ontological economy would seem to suggest that we should admit those and only those distinctions that are needed to explain substitutivity failures. But we have just seen that a pragmatic solution to at least some substitutivity puzzles is inevitable and that, once this style of explanation is available, ultra-fine-grained intensional distinctions are probably not be needed to explain the versions of the substitutivity puzzles for which they were designed. If so, considerations of uniformity and ontological economy would lead us to reject such distinctions. Supposing that this is right, one wonders how far this sort of elimination can go. What types of fine-grained intensional distinctions, if any, survive a systematic attempt to explain substitutivity failures pragmatically?

A transcendental argument yields a partial answer to this question: the fine-grained distinctions that survive must include at least those that are needed to spell out satisfactory pragmatic explanations. It turns out that very fine-grained intensional distinctions are needed for this purpose. Here is the argument.

Consider the person "half-way" along in the process of mastering the predicate 'masticate'. He asserts, "I am sure that whatever masticates chews, but I am not sure that whatever chews masticates." In an actual conversational context the person certainly could assert something true 
by saying this. The problem is to identify what it is. The ultra-finegrained theory, developed in response to the original puzzle above, identifies the proposition that whatever masticates chews and the proposition that whatever chews masticates. The reason is that the two abstracts 'that whatever masticates chews' and 'that whatever chews masticates' have exactly the same grammatical form: $[(\forall x)(1 x \rightarrow 2 x)]$. So this theory implies that the sentence uttered by the person in our present example strictly and literally expresses something that is false. (Indeed, the utterance would express something formally contradictory, namely, that the person is sure of $[(\forall x)(1 x \rightarrow 2 x)]$ and not sure of $[(\forall x)(1 x \rightarrow 2 x)]$.$) Since on the ultra-fine-grained theory the uttered$ sentence expresses something that is strictly and literally false and since our person has asserted something that is true, what he has asserted must be something other than what the sentence strictly and literally expresses. Therefore, the problem of identifying what truth he has asserted cannot be solved semantically; it must be solved pragmatically.

In pragmatics, we may take into account, not only the syntactic and semantic features, but also features of the conversational context and Gricean rules of conversation. Given all this information, one is led to identify the person's true assertion with something like the following:

I am sure that whatever satisfies the predicate 'masticate' also chews, but I am not sure that whatever chews also satisfies the predicate 'masticate'.

This pragmatic solution is a good first try, but there is a problem with it. ${ }^{27}$ (Indeed, there are several philosophers - Tyler Burge, for example - who think this is a fatal problem requiring us to revolutionize our thinking about PRPs and propositional attitudes.) The problem is as follows.

Suppose that the person who utters the above sentence - 'I am sure that whatever masticates chews, but I am not sure that whatever chews masticates' - is a child (or a slow-learning adult) who appears to have no command of metalinguistic concepts we take for granted. In particular, suppose that this child appears to have no articulated concepts from linguistic theory such as the syntactic concept of a linguistic predicate or the semantical concept of satisfaction(-in-English), and suppose that the child appears to be unfamiliar with any device (e.g., quotation) for naming expressions. Furthermore, when we try to teach the child these bits of linguistic theory, he has great difficulty learning them. (He learns to use the new predicate 'masticate' far more readily.)
However, a few years later when we try again to teach him these things, he learns them quickly. This shows, so the worry goes, that the above pragmatic analysis of his assertion represents him as having reached a stage of conceptual development well beyond anything we can plausibly attribute to him (i.e., a stage at which he has command of the indicated concepts from linguistic theory). If so, the pragmatic analysis is mistaken; the child's assertion could not have involved the metalinguistic concepts attributed to him by this analysis.

There appears to be only one general way out of this problem, and that is to treat our child's apparent ignorance of metalinguistic concepts as a species of the special kind of ignorance involved in of the paradox of analysis. Consider two analogies. First, suppose a child can sort variously shaped objects so well that it becomes plain that he recognizes, say, the circular objects as circular and, therefore, that he has command of the concept of circle. However, suppose that the child displays no particular behavior to indicate that he has command of the concept of a (mathematical or physical) point, the concept of a locus of points, the concept of a (mathematical or physical) plane, etc. When we try to teach him geometric theory - with its definition of circle as a locus of points in the same plane equidistant from a common point - we get nowhere. (If he were a few years older, he would be able to learn this readily.) In this situation it is natural to characterize the child as follows, he has an unanalyzed concept of circle, that is, an unanalyzed concept being-a-locus-of-points-in-thesame-plane-equidistant-from-a-common-point. However, he lacks the theoretical concepts (point, locus, plane, etc.) needed to unpack this unanalyzed concept. Accordingly, he lacks the analyzed concept of circle, that is, the analyzed concept of being a locus of points in the same plane equidistant from a common point.

For a second analogy, consider someone who can reliably tell whether a middle-sized object comes to a halt smoothly. But this person seems to have no grasp of the sophisticated concepts of calculus required to say what it is for an object to come to a halt smoothly; indeed, if the person has limited mathematical aptitude he might never be able to grasp the relevant parts of calculus. It would be natural to say of this person that he has an unanalyzed concept of coming-to-a-halt-smoothly but that he lacks the specific theoretical concepts needed to unpack this unanalyzed concept and, accordingly, that he lacks the analyzed concept of coming to a halt smoothly.

With these geometry and calculus examples in mind, let us return to 
the linguistics example. Suppose that a child is not yet in command of various theoretical concepts from linguistics, concepts such as satisfaction-in-English, linguistic predicate, and spelling (or quotation). Despite this, it should still be possible for the child to have an unanalyzed concept whose analysis involves such theoretical concepts from linguistics. This would be quite analogous to the child's having an unanalyzed concept of circle (i.e., an unanalyzed concept of being-a-locus-of-pointsin-the-same-plane-equidistant-from-a-common-point) and yet not being in command of the'theoretical concepts from geometry (point, locus, plane, etc.) needed to unpack this concept. And it would also be analogous to the child's having an unanalyzed concept of coming-to-ahalt-smoothly and yet lacking the theoretical concepts from calculus needed to unpack this concept. Since this sort of thing is commonplace in geometry and calculus examples, surely nothing can prevent it from happening in linguistics examples, too. It would just be dogmatic to rule out this kind of phenomenon. ${ }^{28}$

Let us apply these conclusions to our example. Our child who was "half-way" along in the process of learning to use the predicate "masticate' has an unanalyzed concept of satisfying-the-predicate-'masticate', but he is not in command of the theoretical concepts (satisfaction, predicate, quotation) needed to unpack this unanalyzed concept. If this is right, then we have the makings of a solution to the problem confronting the pragmatic analysis of what true proposition the child asserted when he uttered, "I am sure that whatever masticates chews, but I am not sure that whatever chews masticates." The child's assertion comes to something like this:

I am sure that whatever satisfies-the-predicate-'masticate' also chews, but I am not sure that whatever chews also satisfies-the-predicate-'masticate'.

Since this analysis attributes to the child an unanalyzed concept of satisfying-the-predicate-'masticate', it avoids the problem of mistakenly attributing to the child theoretical concepts that he will acquire only at a more advanced developmental stage. And this is what we needed to avoid the criticism.

Notice, however, that this way of salvaging the pragmatic solution of the original 'chew'/'masticate' substitutivity puzzle is based on a very fine-grained intensional distinction, namely, the distinction between an unanalyzed concept of satisfying-the-predicate-'masticate' and the analyzed theoretical concept of satisfying the predicate 'masticate'. ${ }^{29}$
Now, as far as I can tell, this outcome is unavoidable. There appears to be no way of salvaging the pragmatic solutions to substitutivity puzzles that does not somehow invoke antecedently given intensional distinctions that are very fine-grained. Here, then, is a place where very fine-grained intensional distinctions cannot, even in principle, be eliminated by the technique of pragmatic explanation: they are needed to make the pragmatic explanations satisfactory. If this is right, a very fine-grained intensional logic is inevitable.

\section{SELF-CONSTITUENCY, MUTUAL KNOWLEDGE, AND THE PARADOX OF ANALYSIS}

If fine-grained PRPs are inescapable, what about type-freedom? In the next section I will argue that all types of type freedom are inescapable except for one, namely, self-constituency. The purpose of the present section is to suggest that the leading argument for fine-grained selfconstituency is unsound. Specifically, I will suggest that this argument turns on a certain confusion over the paradox of analysis. (We have just seen that the paradox of analysis is a problem that needs to be solved if any intensional logic is to be able to handle certain very elementary substitutivity problems.)

The argument for fine-grained self-constituency I have in mind alleges that self-constituency is required to account for the phenomenon of mutual knowledge. This argument proceeds as follows. Without selfconstituency, two people with mutual knowledge would have to have infinitely complex thoughts or infinitely many thoughts (perhaps one for each finite number of embeddings plus perhaps one for each limit ordinal to "sum up" all the preceding finite levels). So if there were no self-constituency, a person who thinks that he or she has mutual knowledge with another must have incredibly complex thought(s). But such thought(s) far exceeds an ordinary person's conceptual development. By contrast, a thought involving self-constituency is very simple to articulate: you and I both know this, where one accompanies the utterance of 'this' with an appropriate gesture suggesting "self-reference." Thus, the self-constituency theory is the only satisfactory theory of mutual knowledge. Or so the argument goes.

This argument, however, seems to be question-begging, for it disregards the phenomenon of the paradox of analysis. As we saw in the previous section, the fact that someone does not know calculus hardly shows that calculus is not involved in the analysis (definition) of the 
person's unanalyzed concept of coming-to-a-halt-smoothly. By the same token, suppose that a person does not know the theory of intensional logic, and suppose that the person has no articulate command of propositions with a large number of self-embeddings (i.e., propositions denoted by intensional abstracts that contain several - perhaps infinitely many - iterated occurrences of 'think' or 'know'). This does nothing to show that the person does not think unanalyzed propositions whose analysis (definition) involves a large number of self-embeddings, Likewise, the fact that the person does not know the theory of intensional logic and has no articulate command of propositions with a large number of self-embeddings does nothing to show that self-embedding is not sufficient to analyze (define) the person's unanalyzed concept of mutual knowledge. We have already seen that the fine-grained distinction between unanalyzed and analyzed concepts is needed to solve certain elementary substitutivity puzzles. And it is not at all implausible that this machinery is sufficient for dealing with the phenomenon of mutual knowledge. That is, given the apparatus of unanalyzed concepts, perhaps we can characterize ordinary mutual knowledge as a kind of unanalyzed multiple (perhaps infinite) self-embedding. When we unpack unanalyzed mutual-knowledge propositions, the resulting analyzed propositions will typically be unfamiliar to the people having the mutual knowledge. But all this shows is that ordinary people are not in command of these analyzed propositions. It does not show that they are not in command of the unanalyzed propositions. Hence, the kind of ignorance upon which the mutual-knowledge arguments for self-constituency rest might be nothing more than the kind of ignorance involved in commonplace occurrences of the paradox of analysis. If so, the above argument fails to win its conclusion. It would amount to an elementary confusion regarding the paradox of analysis.

In fact, this ignorance argument for self-constituency might cause trouble for the self-constituency theory itself. Suppose that the selfconstituency theory is right; that is, suppose that there is some mutual knowledge proposition (call it this) such that: this $=[I$ think this $]$. In this case, the following identities would hold: [I think this] $=[\mathrm{I}$ think [I think this $]]=[I$ think $[I$ think $[I$ think this $]]]=[I$ think $[I$ think $[I$ think $[I$ think this] ] ] $=\ldots$ and so on ad infinitum. But is it plausible that, when I was a child with mutual knowledge, I could think:

[I think [I think [I think [I think [I think [I think [I think [I think [I think [I think this]]|]]]]]]].

It hardly seems so. Something seems to have gone wrong.
I think that the most plausible way for self-constituency theorists to deal with this problem is to invoke the machinery of unanalyzed concepts. Specifically, self-constituency theorists would hold that this, the proposition we have been discussing, is an unanalyzed proposition. When this unanalyzed proposition is partially analyzed, its constituents are: me, thinking, and the unanalyzed proposition this. If so, it would follow that this, which is an unanalyzed proposition, cannot be identical to the proposition that would ordinarily be expressed by 'I think this', for the latter proposition would ordinarily be a partially analyzed proposition. (If I were to assert 'I think this', I would ordinarily have an articulated awareness of the constituents: me, thinking, and this.) However, if self-constituency theorists must take this line, they will have no real life examples of analyzed propositions that are constituents of themselves. At best, they will have examples of unanalyzed propositions that are constituents of themselves. But if their best examples are always unanalyzed propositions, it is a theoretical question of how best to analyze these propositions. Self-constituency theorists will have run out of direct evidence supporting their preferred analysis. Accordingly, the issue must be settled on grounds of theoretical virtue. However, it is plausible that the most economical theory is that which denies selfconstituency and instead treats all the problematic examples as unanalyzed propositions whose complete analysis would involve infinitely many self-embeddings but no self-constituency. This theory is more economical than the self-constituency theory, for on the latter theory the problematic examples must also be treated as unanalyzed propositions whose complete analysis would involve infinitely many selfembeddings. So it is not clear that there is any theoretical advantage that justifies positing fine-grained self-constituency. ${ }^{30}$

\section{A TRANSCENDENTAL ARGUMENT FOR FINE-GRAINED} TYPE-FREEDOM

Suppose our goal is to do semantics for natural language. Then the most elegant theory of meaning for predicates, open-sentences, and sentences is provided by a fine-grained type-free theory of PRPs. But many people (Quine, Davidson, etc.) are skeptical about the need for a theory of meaning in a satisfactory comprehensive theory of the world; at most, a semantical theory need only provide a theory of truth and reference. We reply that PRPs are appropriate even in a theory of truth and reference, for in natural language 'that'-clauses and infinitive and 
gerundive phrases call for a theory of truth and reference in which these intensional abstracts refer to PRPs.

Suppose that this kind of PRP semantics provides the most straightforward theory of truth and reference for natural languages containing intensional abstracts. Does it follow that PRPs really exist? Not at all, say our extensionalist critics, for there is a certain kind of possibility we must rule out before we can be sure that PRPs really exist. This possibility goes as follows. It is in principle possible that in the evolution of natural languages crazy sorts of syntactic constructions could emerge. Suppose the most straightforward way to give truth conditions for atomic sentences containing such syntactic constructions is to treat the syntactic constructions as if they refer to some special category of items. That does not show that there really are such items. For, strictly and literally, these atomic sentences might all be degenerately false; or, worse, they all might be neither true nor false. Given the possibility that some special syntactic constructions in natural language are defective in this way, how are we to decide which ones are and which ones are not? We intensionalists should have an argument that intensional abstracts and the associated ontology of PRPs - are not defective in this way.

To see the problem more clearly, consider the following rather fanciful example. (The purpose of the example is to bring out a philosophical point; it need not be likely but only metaphysically possible.) In the sentence 'Someone walks slowly in the plaza' we might associate with the adverb 'slowly' a rate, namely, the rate slow. Similarly, we might associate with the verb 'walks' an kind of action, namely, walking. And we might associate with 'walks slowly' a more specific kind of action, namely, slow walking. We might associate with 'in the plaza' a place, namely, in the plaza. Indeed, although it would be less natural, we might even associate with 'walks in the plaza' another specific kind of action, namely, walking in the plaza. But is there really anything semantically associated with the string 'slowly in the plaza', a new ontological category that we might call rate-places? It hardly seems so. More likely, anyone who accepts rate-places on this dubious basis has just been mislead by syntax into inventing a new metaphysical category that has as yet been given no justification. ${ }^{31}$ Or if rate-places are not ontologically offensive enough for you, consider an alleged metaphysical category of items semantically associated with the string 'slowly in'. (We might call these dubious items rate-relations.) But if no more justification is offered, it would be wrong to posit rate-relations. As far as we can tell, rate-places and rate-relations are not real; they are metaphysical fictions having no real being. Now suppose that for some reason (or, even worse, for no reason besides finding it cute) people begin to "nominalize" phrases like 'slowly in the plaza': 'to be slowly in the plaza', 'being slowly in the plaza', or what not. And people utter such things as 'I like being slowly in the plaza more than being quickly at the ski resort'. Although this course of events would be unlikely, surely it would be (metaphysically) possible. (If you doubt this, do you have an argument that it is metaphysically impossible?) Suppose then that this possibility were realized. In that case it would be a task for philosophers of language and linguists to figure out the semantics for sentences containing these new noun phrases.

Suppose that after much work these theoreticians announce to the scientific and philosophical world that they have actually discovered a new metaphysical category, plates, which are a bit like places and a bit like rates but not reducible to them. Reductionistically inclined people respond by trying to reduce plates to something else, for example, to functions or to sets; anti-reductionists reply in their usual ways. But something has gone seriously wrong here. There are really no such things as plates. If we are seeking a comprehensive theory of the world, we should just leave them out. (Notice the pronoun 'them': how insidious apparent reference to these nonentities can be!) At least this is what plate eliminativists would say. And no one can deny feeling the strong intuitive pull of this response.

The question confronting us proponents of intensional logic and PRPs is this. Do our favorite syntactic constructions - 'that'-clauses and gerundive and infinitive phrases - and our inferred metaphysical categories of fine-grained type-free PRPs have a genesis similar to that of plates? We do not think so, but what reason do we intensionalists have for thinking this? If we cannot show that PRPs and intensional abstraction have a fundamentally different status from that of plates and plate-talk, principles of economy would seem to oblige us to give up the whole business. In this way, we intensionalists must do more than rely on surface features of natural language; we must face head-on the spectre of Quinean extensionalism and of Carnapian ontological conventionalism.

Surely PRPs and intensional abstraction (in contrast to plates and plate-abstracts) do have a privileged and fundamental status, but how are we to show this? I submit that for an answer we ultimately must 
turn to the theory of knowledge. The idea is to give a transcendental argument. The argument consists of two stages. The first is to specify the conditions that, according Quinean extensionalists or Carnapian conventionalists, must be met by any acceptable comprehensive theory of the world; the second is to show that these conditions cannot be met unless the theory invokes PRPs. (Here I am adapting a style of argument developed by George Myro. ${ }^{33}$ In what follows I will concentrate on Quinean extensionalism; minor changes would be needed to meet the challenge of Carnapian conventionalism, but the argument would have substantially the same form.) To justify their own scientific and philosophical theories and to criticize those of their opponents, extensionalists must inevitably invoke a principle of epistemic appraisal or acceptability. The following, which is extrapolated from the writings of Quine, captures what is at the heart of these principles:

(1) A theory is acceptable if and only if it is (or belongs to) the simplest overall theory that explains the data. ${ }^{34}$

When taken together, the natural sciences (plus perhaps extensionai mathematics) comprise the simplest overall theory that explains the data.

It follows from these two principles that a theory is acceptable if and only if it is (or belongs to) the overall theory that consists of the natural sciences (plus perhaps extensional mathematics). Intensional logic and the theory of PRPs does not belong to natural science (or extensional mathematics); therefore, these theories are not acceptable. So argues the extensionalist.

Notice, however, that the expressions 'acceptable', 'simplest', 'explain', and 'data' do not belong to the primitive vocabulary of this overall theory (natural science plus extensional mathematics). Let us suppose that these expressions are not definable in that primitive vocabulary. In this case, the extensionalists' two basic principles - and all the powerful conclusions that depend on them - would not belong to their overall theory and, therefore, would not be acceptable according to extensionalism. This self-defeating consequence can be avoided only if the key expressions 'acceptable', 'simplicity', 'explanation', and 'data' are, contrary to our supposition, definable within the overall theory. For this reason, the extensionalist is committed to the thesis that these expressions are indeed so definable. But this thesis entails that the overall extensionalist theory must possess an apparatus for representing definitional relationships. Now this apparatus either would or would not be metalinguistic. If it were not metalinguistic, it would have to be (something like) one of the following:

By definition, for all $x, F x$ iff $\ldots x \ldots$
It is definitionally true that, for all $x, F x$ iff $\ldots x \ldots$.

The concept of being an $x$ such that $F x=$ the concept of being an $x$ such that . . . . . .

$F$-ness $=$ the property of being an $x$ such that $\ldots x \ldots$

(Universal material biconditionals would not suffice, for they do not have the force of definitions. On the contrary, a universal material biconditional 'For all $x, F x$ iff $\ldots x \ldots$. ' is just an ordinary theoretical sentence about $F$ s.) But constructions of this type clearly have a finegrained intensional logic, and the simplest general explanation of such constructions is in terms of fine-grained intensional entities. So on this option, extensionalists would be forced to invoke a logical construction and an associated ontology that, by their very own principles, is unacceptable. On the other hand, if the apparatus for representing definitional relationships were metalinguistic, it would have to involve a strong semantical term like 'synonymous in $L$ ', 'definitionally equivalent in $L^{\prime}$, 'analytic in $L$ ', or some kindred term. But once again strict extensionalism would be thwarted. For generalized Quinean indeterminacy considerations show that strong semantical terms like 'synonymous in $L$ ' cannot be defined using only terms belonging to the natural sciences and extensional mathematics. On this metalinguistic option, extensionalists would again be committed to a definitional apparatus that, according to the central principles of their philosophy, is unacceptable. This is not to say that strong semantical notions like synonymy cannot be defined. They can be, but only in terms of intentional notions - along Gricean lines, for example. But considerations rather analogous to those leading to Quine's indeterminacy thesis show that these intentional notions cannot be defined using only terms belonging to the natural sciences and extensional mathematics. ${ }^{35}$ At the core of these considerations is the fact that the logic for intentional matters is fine-grained intensional logic. However, the simplest explanation for fine-grained intensionality in logic is in terms of fine-grained intensional entities. So, just as in the case of a non-metalinguistic 
apparatus, a metalinguistic apparatus for representing definitional relationships produces an ontological commitment to fine-grained intensional entities.

Our conclusions so far are the following. Extensionalists are forced by their own basic principles to avail themselves of some apparatus for representing definitional relationships. But any such apparatus - metalinguistic or non-metalinguistic - turns out to be unacceptable according to these very same principles. Thus, extensionalism in its strict form is a self-defeating philosophy and, hence, is unacceptable. Further, since any apparatus adequate for representing definitional relationships presupposes a logical theory that is ontologically committed to fine-grained intensional entities, extensionalists have no choice but to revise their basic principles (1) and (2) in order to make room for this inevitable intensional ontology. ${ }^{36}$

This line of argument shows that a logical theory having a finegrained intensional ontology is unavoidable in any acceptable comprehensive theory, but in the same vein can we show that this logical theory must be type-free? I think we can. We have seen that principles (1) and (2) must be revised in order to avoid self-defeating consequences. Suppose that we have done so. I submit that, by examining the resulting principles, we can show that anyone making an acceptable claim to have an acceptable overall theory is committed to each type of type-freedom (except self-constituency).

To illustrate; let us consider self-embedding. Anyone making an acceptable claim to have an acceptable overall theory needs a theory (or definition) of acceptability that, by its very own standards, is acceptable. That is, the person needs a theory of acceptability:

$\left(1^{\prime}\right) \quad$ A theory is acceptable iff ....

such that the proposition that $\left(1^{\prime}\right)$ is acceptable is a thesis of the overall theory. But the latter proposition involves self-embedding:

It is acceptable that a theory is acceptable iff . . . .

In symbols,

$$
A[(\forall x)(A x \leftrightarrow \ldots x \ldots)] .
$$

In this way, self-embedding seems to be an inevitable feature of an overall theory that, by its own standards, is acceptable. ${ }^{37}$ We thus have a transcendental argument, not just for fine-grained PRPs, but for selfembeddable fine-grained PRPs.
Similar considerations show that the other favored forms of typefreedom - transcendental predicates, universal universal quantification, impredicativity, and self-instantiation - are also inevitable. The upshot is that fine-grained type-free intensionality has a special objective status. Although many syntactic constructions in natural language might, for all we know, commit us to unfounded metaphysical categories. The commitment to fine-grained type-free intensional entities is different. This commitment is transcendentally justified, for to avoid self-defeat we have no choice but to incorporate these basic entities into any acceptable comprehensive theory of the world.

NOTES

'There has also been terminological confusion in use of the terms 'extensional' and 'intensional'. For example, some people talk of "nonextensional sets." But this is an unjustified deviation from traditional usage. An item is an extensional entity if it satisfies the extensionality principles associated with the item's ontological category. In the case of sets, the key extensionality principle is that for all sets $y:(\forall z)(z \in x \cdots$ $z \in y) \rightarrow x=y$. Traditionally, sets by nature satisfy this principle; if an item does not satisfy this principle, set theorists would deem it a nonset. Calling it a "nonextensional set" would count as a simple category mistake in the eyes of traditional set theorists.

Some people have deemed a predicate "intensional" if it is satisfied by the Gödel number of one sentence but not the Gödel numbers of another sentence that is equivalent to this sentence in truth value. But this too deviates from traditional usage, both logico-linguistic and ontological. It does not even match the nonstandard RussellWhitehead usage according to which a propositional function is deemed "intensional" if it is satisfied by a proposition and yet is not satisfied by another proposition that is equivalent to this proposition in truth value.

2 Some transcendental predicates (e.g., 'is self-identical') apply not just across metaphysical categories but to everything one can talk about (in the language of the theory); such predicates express properties that are instances of themselves. But not all transcendental predicates are like this. For example, 'is contemplated by Saint Francis' is transcendental, for the inquisitive saint did, we may be sure, contemplate at least one item from each metaphysical category. However, this predicate does not apply to everything; for, being limited, the saint did not contemplate everything. Nor is it the case that the property expressed by this predicate is an instance of itself, for the humble saint never contemplated the property of being contemplated by Saint Francis. (Consider also transcendental predicates like is a possible object of thought that is not an instance of itself'.) Moreover, not every predicate that expresses a property that is an instance of itself is transcendental. For example, 'is a property' does not apply to relations or propositions, but it does express a property that is an instance of itself.

Incidentally, suppose that some PRP theory contains a transcendental predicate (e.g., ' $x=x^{\prime}$ ') that expresses a self-instantiated property. It does not follow that in the theory this predicate is self-embeddable in the sense I am about to introduce in the text. For even though the sentence ' $[x=x]_{x}$ has $[x=x]_{x}$ ' might be true and/or provable in 
the theory, more complex intensional abstracts involving self-embedding (e.g., the intensional abstract ' $\left[\left[x=\left.x\right|_{x} \text { has }[x=x]_{x}\right]^{\prime}\right.$ ) might not be well-formed terms in the theory.

${ }^{3}$ Nino Cocchiarella, review of Quality and Concept. I. G. McFetridge, review of Quality and Concept.

${ }^{4}$ I prove this and a series of kindred results in "The Significance of Completeness Results in Intensional Logic."

5 Cocchiarella (ibid.) claims that incompleteness can be proven when the first closure condition is imposed. He begins with the premise that an arbitrary first-order necessity sentence ' $\left.N \mid A\left(F_{1}, \ldots, F_{n}\right)\right]$ ' - where ' $F_{1}$ ', $\ldots,{ }^{\prime} F_{n}$ ' are the predicates occurring in $A$ - is true in a model if and only if the second-order sentence ' $\left(\forall F_{1} \ldots F_{n}\right) A$ ' is true in the model. Then he claims that this implicit second-order element in first-order intensional logic is enough to prove incompleteness. But his premise is based on an elementary error. To see why, choose any model in which interpretation I assigns the property of being self-identical to the primitive predicate ' $F$ '. That is, $\mathbf{I}\left({ }^{\prime} F\right.$ ' $)=[x=x]_{x}$. On this interpretation, the intensional abstract ' $[(\exists x) F x \mid$ ' would denote $[(\exists x) x=x]$, i.e., the necessary proposition that something is self-identical. So on this interpretation the first-order intensional sentence ' $N[(\exists x) F x]$ ' is true. However, according to Cocchiarella's premise, this sentence is true on an interpretation if and only if the second-order sentence ' $(\forall F)(\exists x) F x$ ' is true on the interpretation. But this second-order sentence false; indeed, it is logically false. So Cocchiarella's premise does not hold, and his alleged incompleteness proof fails.

The source of Cocchiarella's error is something like this. In PRP semantics, propositions are the primary bearers of necessity; the mere fact that a syntactically simple sentence like ' $(\exists x) F x$ ' is not true by virtue of its syntactic form tells us nothing about whether the proposition expressed by the sentence (i.e., the proposition that $(\exists x) F x$ ) is necessary and, in turn, whether the sentence ' $N[(\exists x) F x]$ ' is true or false in a given model. Cocchiarella's error seem to arise from a kind of generalized use/mention confusion, that the syntactic form of a linguistic expression of a proposition and the modal status of the proposition should match up. But this only happens in very special cases; it does not typically happen.

Incidentally, incompleteness of first-order intensional logic would follow if we had the following premise (which is adapted from an argument that Cocchiarella gives about first-order modal logic): for any first-order extensional sentence ' $A$ ', the first-order intensional sentence ' $N|A|$ ' is true in a model if and only if ' $A$ ' is a logically valid firstorder extensional sentence, i.e., a theorem of first-order extensional logic. Given this premise, if first-order intensional logic were complete, then for every such sentence ' $A$ ', the intensional sentence ' $\neg N[A]$ ' should be valid (and, hence, a theorem) if and only if ' $A$ ' is not a logically valid first-order extensional sentence. But the latter sentences are not recursively enumerable, so the former are not either. Therefore, not every such sentence ' $\neg N[A]$ ' can be a theorem. Consequently, first-order intensional logic is incomplete. However, the premise is based on an elementary error. To see this, let ' $A$ ' be the invalid first-order extensional sentence ' $(\exists x) F x$ ', and consider any model like that discussed a moment ago, wherein interpretation $\mathbf{I}$ assigns to ' $F$ ' the property of being self-identical, i.e., $[x=x]_{x}$. In such a model the intensional abstract ' $[(\exists x) F x]$ ' denotes $[(\exists x) x=x]$, i.e., the necessarily true proposition that something is self-identical. Therefore, in this model the first-order intensional sentence ' $N[(\exists x) F x]$ ' would be true.
However, according to the premise, this sentence should be false because the first-order extensional sentence ' $(\exists x) F x$ ' is not a logically valid sentence. So the premise does not hold, and thus the alleged incompleteness proof fails. Like the earlier erroneous premise, this erroneous premise seems to be based on a kind of generalized use/ mention confusion, that the syntactic form of a linguistic expression of a proposition and the modal status of the proposition should always match up. And of course this is not so.

6 This style of semantics is advocated on pp. 209-210, Quality and Concept.

7 See "The Thesis of Extensionality," section 37, Quality and Concept, for an extended discussion of this important methodological issue.

8 See, e.g., Bealer $(1979,1982)$, pp. 55-57; Zalta (1980); Mönnich (1983).

` See Aczel (1980); Mönnich (1983); Chierchia and Turner (1988), Aczel (1987), Turner (1987). Some of the following reasons I will give against the propositionalfunction thesis were reasons that originally guided me toward the algebraic approach in Bealer (1979 and 1982). However, some of these reasons have occurred to me afterward.

10 A propositional-function theorist might reply that this argument is an instance of the "fallacy of incomplete analysis." However, this reply is theoretically weak, for it forces the propositional-function theorist to hold that our intuitions here cannot be taken at face value. But other things being equal, a theory is superior if is can take relevant intuitions at face value. Our theory that properties are not propositional functions permits us to do just this.

11 Fine-grained intensions are not needed just for treating the propositional attitudes. They are also needed for treating various purely logical matters such as logical truth and analyticity for propositions. This point is discussed in section 1 of G. Bealer, "The Logical Status of Mind."

12 See Bealer (1979) and chapter 2 (1982).

13 See Church (1954)

14 See Church $(1951,1974)$. See also Anderson (1980).

is This principle is Russellian in flavor. Fregeans would wish to modify it slightly, However, when the appropriate Fregean principles are substituted, we still can make much the same argument against the propositional-function thesis.

${ }^{16}$ C. Anthony Anderson (1986) has independently given a somewhat similar argument in connection with a comparison of Russellian and Fregean higher-order intensional logics.

17 More colloquially, being someone who rajneeshes = being someone who follows rajneesh, and being someone who fondalees = being someone whom Jane Fonda follows. Or to rajneesh $=$ to be someone who follows Rajneesh, and to fondalee $=$ to be someone whom Jane Fonda follows. In the symbolism in Quality and Concept, $|R x|_{\mathrm{x}}=$ $[F x r]_{x}$ and $[F y]_{y}=[F j y]_{y}$.

18. This argument is given in a broadly Russellian setting in which the relevant functions may be applied to individuals, for example, to Jane Fonda and Rajneesh. But much the same argument can be given in a Fregean setting in which the relevant propositional functions are instead applied to individual concepts, for example, to the individual concept of (being) Jane Fonda and the individual concept of (being) Rajneesh.

Some might wonder whether, in the Russellian setting, the problem might not turn on the use of names in intensional contexts. This is not so, for the entire argument can 
be given using externally quantifiable free variables instead of names, Where $u=$ Jane Fonda and $v=$ Rajneesh,

$$
\begin{aligned}
& {[R x]_{x}=[F x]_{x} .} \\
& (\lambda x)(R x)=\mid R x]_{x} . \\
& (\lambda x)(F x v)=[F x]_{x} .
\end{aligned}
$$

Therefore,

$$
(\lambda x)(R x)=(\lambda x)(F x v)
$$

So:

$$
(\lambda x)(R x)(u)=(\lambda x)(F x u)(u) .
$$

Hence, by $(C)$ and $(E)$,

$$
[R u]=[F u v] .
$$

Similarly,

$$
\begin{aligned}
& {[F y]_{y}=\left[\left.F u y\right|_{y} .\right.} \\
& (\lambda y)(F y)=[F y]_{y} . \\
& (\lambda y)(\text { Fuy })=[\text { Fuy }]_{y} .
\end{aligned}
$$

Therefore,

$$
(\lambda y)(F y)=(\lambda y)(F u y) .
$$

So:

$$
(\lambda y)(F y)(v)=(\lambda y)(F u y)(v) .
$$

Hence, by $(\mathrm{C})$ and $(\mathrm{E})$,

$$
[F v]=[F u v] .
$$

Combining these two results, we get:

$$
[R u]=|F v| .
$$

That is,

The proposition that $u$ rajneeshes $=$ the proposition that $v$ fondalees.

However, it seems possible that someone could be consciously and explicitly thinking that $u$ rajneeshes while not consciously and explicitly thinking that $v$ fondalees.

19 If we wish a PRP theory that makes even finer-grained intensional distinctions (for example, so that $[R j] \neq[$ Fjr $]$ ), we should adopt the apparatus introduced in Chapter 3 of Quality and Concept for resolving the paradox of analysis.

Incidentally, some people have suggested that the fondalee/rajneeshee example is a counterexample to the principle of $\beta$-conversion from $\lambda$-calculus. If this were right, so much the worse for $\lambda$-calculus. However, $\beta$-conversion does not seem to be what the example calls into question. How could it? On the standard interpretation of $\lambda$-calculus ' $f(x)$ ' denotes the value that results when function $f$ is applied to argument $x$, where $f$ is assigned to ' $f$ ' and $x$ is assigned to ' $x$ '; and ' $(\lambda x)(f(x))(x)^{\prime}$ ' denotes the value that results where a certain function is applied to argument $x$, where that function is one that yields $f(x)$ as its value when it is applied to argument $x$. Accordingly, given an assignment to ' $f$ ' and ' $x$ ', ' $f(x)$ ' and ' $(\lambda x)(f(x))(x)$ ' cannot fail to denote the same thing. And this generalizes. Therefore, $\beta$-conversion seems unassailable. So the counterexample does not call into question $\beta$-conversion; rather, it calls into question the identification of properties with propositional functions. There is nothing wrong with $\lambda$-calculus. The point is that $\lambda$-calculus cannot be applied as a theory of properties, for properties and propositional functions behave differently.

20 We need not take a stand here on the relationship between unanalyzed intensions and coarse-grained intensions, even though it would be intriguing to explore the prospect of identifying them. For the purposes of the present argument, it is enough that there should be intuitive and/or theoretical grounds (independent of the paradox of analysis) for positing the existence of coarse-grained intensions. Such grounds are spelled out more in fuller detail in Chapters 8-10 of Quality and Concept (especially Section 40). See also David Lewis, "New Work for a Theory of Universals," and section 1 of G. Bealer, "The Logical Status of Mind."

${ }^{21}$ A further problem confronts propositional-function theories that make use of a fundamental logical operation of abstraction to represent how various "complex" properties are "formed." On this approach, one first "forms" 0-ary intensions by simply applying given propositional functions to arguments. Then, to "form" various "complex" properties, one applies the abstraction operation to these 0 -ary intensions indexed to some argument. For example, let $p=$ the fine-grained proposition that this closed plane figure has 3 sides. Then abst $(p, 3)=$ the property of being an $x$ such that this closed plane figure has $x$ sides. For another example, let us define a cegree to be a unit of 60 degrees. (So just as trilaterals have 180 degrees, they have 3 cegrees; just as quadrilaterals have 360 degrees, they have 6 cegrees, etc.) Let $q=$ the fine-grained proposition that this closed plane figure has 3 cegrees. Then abst $(q, 3)=$ the property of being an $x$ such that this closed plane figure has $x$ cegrees. Since $p$ and $q$ are fine-grained, $\operatorname{abst}(p, 3) \neq \operatorname{abst}(q, 3)$. This is the right outcome.

This "abstraction approach," however, cannot be used to treat coarse-grained intensions. To see what the problem is, let $p^{\prime}$ and $q^{\prime}$ be the conditions in the world to which $p$ and $q$ correspond, that is, the condition that this closed plane figure has 3 sides and the condition that this closed plane figure has 3 cegrees. Since $p^{\prime}$ and $q^{\prime}$ are necessarily equivalent, $p^{\prime}=q^{\prime}$. Therefore, no matter how abst is characterized for coarse-grained intensions, abst $\left(p^{\prime}, 3\right)=\operatorname{abst}\left(q^{\prime}, 3\right)$. But, then, which of the following properties should be identified with abst $\left(p^{\prime}, 3\right)$ : the property of being an $x$ such this closed plane figure has $x$ sides, or the property of being an $x$ such that this closed plane figure has $x$ cegrees? (Note that these properties are not necessarily equivalent.) The choice is arbitrary. Furthermore, what happens to the property that is not chosen to be abst $\left(p^{\prime}, 3\right)$; how does it get "formed" on this approach? Evidently, there is no answer.

22 A binary relation $r$ is univocal iff for all $x, y, z$, if $x, y$ stand in relation $r$ and $x, z$ stand in relation $r$, then $y=z$.

23 This general approach to substitutivity failures is discussed in detail in section 39 "Pragmatics," Quality and Concept; a concrete example of the conversational pragmatics is traced out on pp. 172-174. A similar approach has recently been proposed by Nathan Salmon in Frege's Puzzle.

${ }^{24}$ Hilary Putnam, "Synonymity and the Analysis of Belief Sentences." 
${ }^{25}$ In the semantics, for example, we define a new type of model structure in which there is a primitive logical operation for each different syntactic form. These operations will be $1-1$; their ranges will be disjoint, and their behavior with respect to the extension functions $H$ in $K$ will be just what one would expect.

26 Benson Mates, "Synonymity."

27 Tyler Burge expresses a closely related worry; see pp. $127 \mathrm{ff}$. , 'Belief and Synonymy'; p. 97, 'Individualism and the Mental', and 'On Knowledge and Convention'. The issue here dramatizes the fact that any adequate theory of language learning must incorporate a resolution of the paradox of analysis.

${ }^{28}$ But this is what Tyler Burge seems to be saying ibid.

${ }^{29}$ A formal semantics that deals with fine-grained distinctions like this may be developed along the lines suggested in Quality and Concept, p. 257, n. 17.

The only other known way of trying to solve this sort of substitutivity puzzle is by treating the standard propositional attitudes as three-place relations holding among a person, a proposition, and a "mode of presentation." However, there are a host of problems with this proposal. For example, it runs into trouble with iterated propositional attitudes, quantifying-in, and general sentences that mix intentional and nonintentional predicates. We plan to spell out these difficulties in a future publication.

16) In his theory of intentional causation, John Searle maintains that self-constituency is involved in perception reports and intentional action reports. (See chapter 4 "Intentional Causation," Intentionality.) But Searle's arguments ride on the same confusions over the paradox of analysis made by the self-constituency theorist described in the text.

31 Of course, there might be some kind of function indirectly associated with 'slowly in the plaza'. However, unlike rate-places, functions do not comprise a new metaphysical category; they are just a kind of relation, namely, univocal relations. But "rate-place" realists are not advocating functions; they are advocating a new metaphysical category above and beyond functions.

32 Another sort of example might help. When taking notes, I often engage in certain sloppy practices - "private language," if you will. For example, instead of using intensional abstracts ('that'-clauses and gerundive and infinitive phrases), I often use expressions that on the surface appear to be quotation names; sometimes I even use external quantifiers to "bind" variables occurring within these "quasi-quotation-names." Although my impression is that this sloppiness occurs randomly, there might for all I know be some pattern to it. This might lead an observer to conclude that these quasiquotation-names actually purport to refer to items that are neither PRPs nor linguistic expressions but rather are items in special new metaphysical category. Now suppose that my note-taking practices were to become fashionable: everyday people begin to write in this way all the time, and they develop what seem to be linguistic intuitions about how properly to use these quasi-quotation-names. These expressions appear to be neither genuine intensional abstracts nor genuine quotation names. We would then have a case of a syntactic construction that on the surface appears to generate an ontological commitment to a previously unknown metaphysical category. Would you be justified in concluding on the basis of this linguistic data that there really are items belonging to this previously unknown metaphysical category? Surely not. The question to us intensionalists is this: what reason do we have for thinking that intensional abstracts and PRPs have a fundamentally different status?

${ }_{33}$ George Myro, "Aspects of Acceptability." The present adaptation of George Myro's argument is given more fully in of my paper "The Logical Status of Mind." In the latter paper, a number of other supporting arguments are also given; see especially Sections 1 and 5 .

34 In place of this principle, an extensionalist might adopt a coherentist principle. The outcome would be the same, however. For more on coherentism, see Section 7 "Coherentism" in my paper "The Philosophical Limits of Scientific Essentialism."

${ }^{35}$ For a full argument, see G. Bealer, "Mind and Anti-Mind: Why Thinking has no Functional Definition."

${ }^{36}$ There is a somewhat different transcendental argument for the same conclusion. In this argument, we focus not on definitions but rather on the notion of data. Specifically, we argue that the only satisfactory general theory of what counts as data relies on finegrained intensionality. Given this conclusion and given the extensionalists' commitment to principle (1), they have no choice but to admit fine-grained intensionality. So once again, extensionalism turns out to be a self-defeating philosophy. This argument is spelled out in Section 5 of "The Logical Status of Mind."

${ }_{37}$ Such a theory must include a type-free solution to the Montague-Kaplan paradox. In "Propositions and Representations" Nicholas Asher and Hans Kamp have made much progress on this difficult problem. Although their solution is not entirely perfect, it does provide strong evidence that our transcendental demand for a theory that, by its very own standards, is acceptable is a demand that can be met.

\section{REFERENCES}

Asher, N. and Kamp, H.: 1987, 'Self-Reference, Attitudes and Paradox'. This volume.

Aczel, P.: 1980, 'Frege Structures and the Notions of Proposition, Truth and Set', in

Keisler, J., Barwise, J., and Kunen, K. (eds.), The Kleene Symposium, North Holland, Amsterdam, pp. 31-59.

Aczel, P.: 1987, 'Algebraic Semantics for Intensional Logics I'. This volume.

Anderson, C. A.: 1980, 'Some New Axioms for the Logic of Sense and Denotation: Alternative (0)', Noûs 14, pp. 217-234.

Anderson, C. A.: 1986, 'Intensional Logic'. Lecture at the American Philosophical Association Central Division Meeting.

Bealer, G.: 1979, 'Theories of Properties, Relations, and Propositions', The Journal of Philosophy 76, pp. 638-648.

Bealer, G.: 1982, Quality and Concept, Clarendon Press, Oxford.

Bealer, G.: 1983, 'Completeness in the Theory of Properties, Relations, and Propositions', The Journal of Symbolic Logic 48, pp. 415-426.

Bealer, G.: 1984, 'Mind and Anti-Mind: Why Thinking has no Functional Definition', Midwest Studies in Philosophy 9, pp. 283-328.

Bealer, G.: 1986, 'The Logical Status of Mind', Midwest Studies in Philosophy 10, pp. 231-274.

Bealer, G.: 1987, 'The Philosophical Limits of Scientific Essentialism', Philosophical Perspectives 1, pp. 289-365.

Bealer, G.: 1987 (manuscript), 'The Significance of Completeness Results in Intensional Logic'.

Burge, T.: 1975, 'Knowledge and Convention', The Philosophical Review 84, pp. 249255 
Burge, T.: 1978, 'Belief and Synonymy', The Journal of Philosophy 75, pp. 119-138

Burge, T.: 1979, 'Individualism and the Mental', Midwest Studies in Philosophy 4, pp. 73-122.

Chierchia, G. and Turner, R.: 1988 (forthcoming), 'Semantics and Property Theory', Linguistics and Philosophy.

Church, A.: 1951, 'A Formulation of the Logic of Sense and Denotation', in Henle, P., Kallen, H. M., and Langer, S. K. (eds.), Structure, Method, and Meaning: Essays in Honor of Henry M. Scheffer, New York, pp. 3-24.

Church, A.: 1954, 'Intensional Isomorphism and the Identity of Belief', Philosophical Studies 5, pp. 65-73.

Church, A.: 1973, 'Outtine of a Revised Formulation of the Logic of Sense and Denotation', Part 1, Noûs 7, pp. 24-33.

Church, A.: 1974, 'Outline of a Revised Formulation of the Logic of Sense and Denotation', Part 2, Noûs 8, pp. 135-156.

Cocchiarella, N.: 1985, Review of Bealer, G. Quality and Concept, in The Journal of Symbolic Logic 50, pp. 254-256.

Cresswell, M. J.: 1985, Structured Meanings, Bradford, Cambridge.

Lewis, D.: 1983, 'New Work for a Theory of Universals', Australasian Journal of Philosophy 61, pp. 343-377.

Mates, B.: 1950, 'Synonymity', University of California Publications in Philosophy 25, pp. 201-226.

McFetridge, I. G.: 1984, Review of Bealer, G. Quality and Concept in Mind 93, pp. 455-458.

Mönnich, Uwe: 1983, 'Towards a Calculus of Concepts as a Semantical Metalanguage', in Bäuerle, R. Schwarze, C. and von Stechow, A., Meaning, Use, and Interpretation of Language, Walter de Gruyter, New York, pp. 1-35.

Myro, G.: 1981, 'Aspects of Acceptability', Pacific Philosophical Quarterly 62, pp. $107-117$.

Putnam, H.: 1954, 'Synonymity and the Analysis of Belief Sentences', Analysis 14, pp. $114-122$.

Salmon, N.: 1986, Frege's Puzzle, Bradford, Cambridge.

Schiffer, S.: 1987, Remnants of Meaning, Bradford, Cambridge.

Searle, J. R.: 1983, Intentionality, The University Press, Cambridge.

Soames, S.: 1985, 'Direct Reference and Propositional Attitudes', Lecture at the American Philosophical Association Pacific Division Meeting.

Turner, R.: 1987, 'Two Issues in the Foundations of Semantic Theory'. This volume.

Zalta, E. N.: 1980, An Introduction to a Theory of Abstract Objects, Ph.D. Dissertation, University of Massachusetts, Amherst.

Dept. of Philosophy,

Reed College, Portland, OR 97202, U.S.A.

Seminar für Naturlich-sprachliche Systeme

Universität Tübingen, 7400 Tübingen, West Germany

\section{SEMANTIC TYPE-CHANGE AND SYNTACTIC RECOGNITION}

\section{FLEXIBLE CATEGORIAL GRAMMAR}

The standard categorial grammar of Ajdukiewicz and Bar-Hillel was based on fixed categories, which could be combined by a rule of functional application. Recently, more flexible versions have been proposed, incorporating rules of category or type change in the process of evaluating expressions. A prominent example is the 'Geach Rule':

$$
\text { change type }(a, b) \text { to }((c, a),(c, b)) \text {; }
$$

which can be used, e.g., to lift sentence negation (type $(t, t))$ to predicate negation (type $((e, t),(e, t)))$. Another well-known case is the 'Montague Rule':

$$
\text { change type } a \text { to }((a, b), b) \text { : }
$$

which can lift proper names (type $e$ ) to noun phrases (type $((e, t), t)$ ). But also, Partee and Rooth 1983 have a rule of 'argument lowering', whose general pattern is this:

$$
\text { change type }(((a, b), b), c) \text { to }(a, c) \text {. }
$$

This changes, e.g., complex intransitive verbs (type $((e, t), t), t)$ to simple predicates (type $(e, t))$.

The general linguistic motivation for type-shifting is manifold (witness the various contributions in Oehrle, Bach and Wheeler (1988) or Buszkowski, Marciszewski and Van Benthem (1988)). For instance, the Geach Rule allows constituent structures in which a function combines with not just its 'completed' arguments, but also with 'parametrized' versions thereof. Such structures have been used to represent discontinuous long-distance dependencies in syntax: a phenomenon which was formerly believed to be an insuperable obstacle to categorial description. (Similar applications have been found in compositional morphology.) Another kind of motivation has been a desire to simplify the baroque category/type system of Montague Grammar. With a regime of type-shifting rules, initial types for expressions can be kept simple, 